\title{
Estimates for Unimodular Multipliers on Modulation Hardy Spaces
}

\author{
Jiecheng Chen, ${ }^{1}$ Dashan Fan, ${ }^{2}$ Lijing Sun, ${ }^{2}$ and Chunjie Zhang ${ }^{3}$ \\ ${ }^{1}$ Department of Mathematics, Zhejiang Normal University, Jinhua 321004, China \\ ${ }^{2}$ Department of Mathematics, University of Wisconsin-Milwaukee, Milwaukee, WI 53201, USA \\ ${ }^{3}$ School of Science, Hangzhou Dianzi University, Hangzhou 310016, China
}

Correspondence should be addressed to Chunjie Zhang; purezhang@hdu.edu.cn

Received 23 November 2012; Accepted 23 January 2013

Academic Editor: Baoxiang Wang

Copyright (C) 2013 Jiecheng Chen et al. This is an open access article distributed under the Creative Commons Attribution License, which permits unrestricted use, distribution, and reproduction in any medium, provided the original work is properly cited.

It is known that the unimodular Fourier multipliers $e^{i t|\Delta|^{\alpha / 2}}, \alpha>0$, are bounded on all modulation spaces $M_{p, q}^{s}$ for $1 \leq p, q \leq \infty$. We extend such boundedness to the case of all $0<p, q \leq \infty$ and obtain its asymptotic estimate as $t$ goes to infinity. As applications, we give the grow-up rate of the solution for the Cauchy problems for the free Schrödinger equation with the initial data in a modulation space, as well as some mixed norm estimates. We also study the $M_{p_{1}, q}^{s} \rightarrow M_{p_{2}, q}^{s}$ boundedness for the operator $e^{i t|\Delta|^{\alpha / 2}}$, for the case $0<\alpha \leq 2$ and $\alpha \neq 1$. Finally, we investigate the boundedness of the operator $e^{i t|\Delta|^{\alpha / 2}}$ for $\alpha>0$ and obtain the local well-posedness for the Cauchy problem of some nonlinear partial differential equations with fundamental semigroup $e^{i t|\Delta|^{\alpha / 2}}$.

\section{Introduction}

A Fourier multiplier is a linear operator $H_{\mu}$ whose action on a test function $f$ on $\mathbb{R}^{n}$ is formally defined by

$$
H_{\mu} f(x)=\frac{1}{(2 \pi)^{n}} \int_{\mathbb{R}^{n}} \mu(\xi) \widehat{f}(\xi) e^{i 2 \pi \xi \cdot x} d \xi .
$$

The function $\mu$ is called the symbol or multiplier of $H_{\mu}$.

In this paper, we will study the unimodular Fourier multipliers with symbol $e^{i t|\xi|^{\alpha}}$ for $t \in \mathbb{R}_{+}$. They arise when one solves the Cauchy problem for dispersive equations. For example, for the solution $u(t, x)$ of the Cauchy problem

$$
\begin{gathered}
i \partial_{t} u+|\Delta|^{\alpha / 2} u=0, \quad(t, x) \in \mathbb{R}_{+} \times \mathbb{R}^{n}, \\
u(0, x)=u_{0}(x)
\end{gathered}
$$

we have the formula $u(t, x)=\left(e^{i t|\Delta|^{\alpha / 2}} u_{0}\right)(x)$. Here $\Delta=$ $\Delta_{x}$ is the Laplacian and $e^{i t|\Delta|^{\alpha / 2}}$ is the multiplier operator with symbol $e^{i t|\xi|^{\alpha}}$ (see [1] for its definition). The cases $\alpha=$ $1,2,3$ are of particular interest because they correspond to the (half-) wave equation, the Schrödinger equation, and (essentially) the Airy equation, respectively.

Unimodular Fourier multipliers generally do not preserve any Lebesgue space $L^{p}$, except for $p=2$. The $L^{p}$-spaces are not the appropriate function spaces for the study of these operators and the so-called modulation spaces are good alternative classes for the study of unimodular Fourier multipliers. The modulation spaces $M_{p, q}^{s}\left(\mathbb{R}^{n}\right)$ were first introduced by Feichtinger [2-4] to measure smoothness of a function or distribution in a way different from $L^{p}$ spaces, and they are now recognized as a useful tool for studying pseudodifferential operators [5-7]. We will recall the precise definition of modulation spaces in Section 2 below.

Recently, the boundedness of unimodular Fourier multipliers $e^{i t|\Delta|^{\alpha / 2}}$ on the modulation spaces has been investigated in $[1,8-15]$. Particularly, one has the following results.

Theorem A (see [11]). Let $s \in \mathbb{R}, 1 \leq p, q \leq \infty, \alpha>1 / 2$ and $\alpha \neq 1$. One has, for $t \geq 1$,

$$
\left\|e^{i t|\Delta|^{\alpha / 2}} f\right\|_{M_{p, q}^{s}} \preceq t^{n|1 / 2-1 / p|}\|f\|_{M_{p, q}^{s+\gamma}}+\|f\|_{M_{p, q}^{s}}
$$


where

$$
\gamma=n(\alpha-2)\left|\frac{1}{2}-\frac{1}{p}\right| .
$$

Here (and throughout this paper), we use the notation $A \preceq B$ to mean that there is a positive constant $C$ independent of all essential variables such that $A \leq C B$.

Theorem B (see [15]). Let $0<p<1,0<q \leq \infty, \alpha>$ $n(1 / p-1)$ and $s \in \mathbb{R}$. Then $e^{i t|\Delta|^{\alpha / 2}}$ is bounded from $M_{p, q}^{s}$ to $M_{p, q}$ if and only if

$$
s \geq \max \{0, \alpha-2\} n\left(\frac{1}{p}-\frac{1}{2}\right) .
$$

In this paper, we use a different method from [15] to prove the following theorem, which, in particular, uses the modulation Hardy spaces $\mu_{p, q}^{s}$ that will be later defined in Section 2.

Theorem 1. Let $s \in \mathbb{R}, 0<q \leq \infty, t \geq 1$. For a positive $\alpha \neq 1$, denote $\gamma=n(\alpha-2)|1 / p-1 / 2|$. Let $\alpha_{0}=2 /(2+n)$ if $n$ is even and $\alpha_{0}=3 /(3+n)$ if $n$ is odd.

(i) Assume $\alpha>\alpha_{0}$. If $p>n /(n+\alpha)$ and $n \geq 2$, one has

$$
\left\|e^{i t|\Delta|^{\alpha / 2}} f\right\|_{M_{p, q}^{s}\left(\mathbb{R}^{n}\right)} \preceq t^{n|1 / p-1 / 2|}\|f\|_{M_{p, q}^{s+\gamma}\left(\mathbb{R}^{n}\right)}+\|f\|_{M_{p, q}^{s}\left(\mathbb{R}^{n}\right)} .
$$

Particularly, the above inequality holds for all $p>0$ if $\alpha$ is a positive even number.

(ii) For any $0<p \leq \infty$, one has

$$
\left\|e^{i t|\Delta|^{\alpha / 2}} f\right\|_{M_{p, q}^{s}\left(\mathbb{R}^{n}\right)} \preceq t^{n|1 / p-1 / 2|}\left(\|f\|_{M_{p, q}^{s+\gamma}\left(\mathbb{R}^{n}\right)}+\|f\|_{\mu_{p, q}^{s-\delta}\left(\mathbb{R}^{n}\right)}\right),
$$

for any $\delta \geq 0$.

Here

$$
\gamma=n(\alpha-2)\left|\frac{1}{p}-\frac{1}{2}\right| .
$$

(iii) Assume $n=1$. If $\alpha>1 / 2$, then

$$
\left\|e^{i t|\Delta|^{\alpha / 2}} f\right\|_{M_{p, q}^{s}(\mathbb{R})} \preceq t^{|1 / p-1 / 2|}\|f\|_{M_{p, q}^{s+\gamma}(\mathbb{R})}+\|f\|_{M_{p, q}^{s}(\mathbb{R})}
$$

for all $p>2 / 3$.

We want to make a few remarks on Theorem 1. First, (iii) in Theorem 1 says that when $n=1$, compared to the case $n \geq 2$ in (i), one obtains a larger range of $\alpha$ and a smaller range of $p$. We do not know if there is a unified formula regarding $\alpha$ and $p$ for all dimension $n \geq 1$. Second, in the proof we will see that, in the low frequency parts of the definition of $M_{p, q}^{s}$, the fractional Schrödinger semigroup has a growth $t^{n|1 / p-1 / 2|}$ when $t$ is growing, but it gains an arbitrary regularity. In the high frequency part, the semigroup can be controlled by

$$
\max \left\{1,\left(t|k|^{\alpha-2}\right)^{n|1 / p-1 / 2|}\right\}
$$

at each piece of its decomposition with frequency $k$. This phenomenon was also more precisely observed in $[1,15]$ (see also [11]). Thirdly, the case $\alpha=1$ was studied in $[8,16]$.

Since the $L^{p}$ norm is dominated by the $H^{p}$ norm and the Riesz transforms are bounded on $H^{p}$, by the Riesz transform characterization of the $H^{p}$ (see Section 2), we easily obtain the following corollary.

Corollary 2. Let $s \in \mathbb{R}, 0<p, q \leq \infty$ and $\alpha>0, \alpha \neq 1$. One has for $t \geq 1$

$$
\left\|e^{i t|\Delta|^{\alpha / 2}} f\right\|_{\mu_{p, q}^{s}} \preceq t^{n|1 / p-1 / 2|}\|f\|_{\mu_{p, q}^{s+\gamma}}
$$

where

$$
\gamma=n(\alpha-2)\left|\frac{1}{p}-\frac{1}{2}\right| .
$$

Our next result shows that the asymptotic factor $t^{n|1 / p-1 / 2|}$ in Theorem 1 is the best for all $p>0$, at least for $\alpha=2$.

Theorem 3. Let $\alpha=2$. The asymptotic factor $t^{n|1 / p-1 / 2|}$ in Theorem 1 is the best. Precisely, for $t>1$, if

$$
\left\|e^{i t|\Delta|} f\right\|_{M_{p, q}^{s}} \preceq t^{\delta}\|f\|_{M_{p, q}^{s}},
$$

then

$$
\delta \geq n\left|\frac{1}{p}-\frac{1}{2}\right|
$$

In the next theorem, we state some mixed norm estimates.

Theorem 4. Let $0<\alpha \leq 2$ and $\alpha \neq 1$. For $0<p_{1} \leq 1 \leq$ $p_{2} \leq \infty$, suppose $n r\left(1 / 2-1 / p_{2}\right)>1$.

(i) If $r \geq q$, then

$$
\|\| e^{i t|\Delta|^{\alpha / 2}} f\left\|_{M_{p_{2}, q}^{s} \|_{L^{r}(0, \infty)}} \preceq\right\| f \|_{M_{p_{1}, q}^{s+(2-\alpha) / r} .}
$$

(ii) If $r \leq q$, then

$$
\|\| e^{i t|\Delta|^{\alpha / 2}} f\left\|_{M_{p_{2}, q}^{s}}\right\|_{L^{r}(0, \infty)} \leq\|f\|_{M_{p_{1}, r}^{s+(2-\alpha) / r}} .
$$

We consider the following linear Cauchy problem with negative power:

$$
\begin{gathered}
i \partial_{t} u+\left|\Delta_{x}\right|^{-\alpha / 2} u=0, \quad(t, x) \in \mathbb{R}_{+} \times \mathbb{R}^{n}, \alpha>0, \\
u(0, x)=u_{0}(x) .
\end{gathered}
$$

We give the grow-up rate of the solution to the above Cauchy problem in the modulation spaces. 
Theorem 5. Assume $\alpha>0$ and $\gamma=n(\alpha+2)|1 / 2-1 / p|$.

(i) Let $1 \leq p<2$. One has that for any $0<r<n p /(n+$ $\alpha([n / 2]+1)(2-p))$

$$
\begin{aligned}
\|u(t)\|_{M_{p, q}^{s}\left(\mathbb{R}^{n}\right)} \preceq & (1+t)^{([n / 2]+1)|2 / p-1|}\left\|u_{0}\right\|_{\mu_{r, q}^{s-\gamma}\left(\mathbb{R}^{n}\right)} \\
& +\left\|u_{0}\right\|_{M_{p, q}^{s}\left(\mathbb{R}^{n}\right)} .
\end{aligned}
$$

(ii) For any $r \leq 2 \leq p$, one has

$$
\|u(t)\|_{M_{p, q}^{s}\left(\mathbb{R}^{n}\right)} \preceq\left\|u_{0}\right\|_{\mu_{r, q}^{s}\left(\mathbb{R}^{n}\right)}+(1+t)^{n|1 / p-1 / 2|}\left\|u_{0}\right\|_{M_{p, q}^{s-\gamma}\left(\mathbb{R}^{n}\right)} .
$$

Now, we study the following Cauchy problem of the nonlinear dispersive equations (NDE):

$$
\begin{gathered}
i \frac{\partial u}{\partial t}-|\Delta|^{-\alpha / 2} u+F(u)=0, \\
u(0, x)=u_{0}(x),
\end{gathered}
$$

where $F(u)=|u|^{2 k} u$ for some positive integer $k$. For $T>0$, the space $Y=C\left([0, T], M_{p, 1}^{s}\right)$ is defined by

$$
\begin{aligned}
C & \left([0, T], M_{p, 1}^{s}\right) \\
& =\left\{u(t, x):\|u\|_{C\left([0, T], M_{p, 1}^{s}\right)}=\sup _{0 \leq t \leq T}\|u(t, \cdot)\|_{M_{p, 1}^{s}}<\infty\right\} .
\end{aligned}
$$

We obtain the quantitative forms about the solution to the above Cauchy problem of the nonlinear dispersive equations.

Theorem 6. Let $2>p>1, \gamma=n(\alpha+2)|1 / 2-1 / p|, \alpha>0$ and assume

$$
\frac{n p}{n+\alpha([n / 2]+1)(2-p)}>1 .
$$

Assume $u_{0} \in M_{r, 1}^{s-\gamma} \cap M_{p, 1}^{s}$ for any

$$
1<r<\frac{n p}{n+\alpha([n / 2]+1)(2-p)} .
$$

There exists $T>0$ such that the above Cauchy system (NDE) has a unique solution $u \in C\left([0, T], M_{p, 1}^{s}\right)$, where $T$ depends on the norm $\left\|u_{0}\right\|_{M_{r, 1}^{s-\gamma}}$ and $\left\|u_{0}\right\|_{M_{p, 1}^{s}}$.

According to the inclusions of modulation space (see Proposition 2.5 in [13]), we know the space of initial data $M_{r, 1}^{s-\gamma} \cap M_{p, 1}^{s}=M_{r, 1}^{s-\gamma}$ if $r \leq p$.

Theorem 7. Let $p \geq 2, \gamma=n(\alpha+2)|1 / 2-1 / p|$. Assume

$$
\frac{p}{2 k+1} \geq 1
$$

and $u_{0} \in M_{r, 1}^{s}$ for any

$$
\frac{p}{2 k+1} \leq r<2
$$

There exists $T>0$ such that the above Cauchy system (NDE) has a unique solution $u \in C\left([0, T], M_{p, 1}^{s}\right)$, where $T$ depends on the norm $\left\|u_{0}\right\|_{M_{r, 1}^{s}}$.

The rest of the paper is organized as follows. In Section 2, we recall or establish some necessary lemmas and known results. Sections 3 and 4 are devoted to the proofs of Theorems 1 and 3, respectively. Finally, in Section 5, we give some applications including the $M_{p_{1}, q}^{s} \rightarrow M_{p_{2}, q}^{s}$ boundedness for the operator $e^{i t|\Delta|^{\alpha / 2}}$ in the case $\alpha \leq 2$ and $\alpha \neq 1$, including negative $\alpha$.

\section{Preliminaries}

2.1. The Definitions. The modulation space is originally defined by Feichtinper in 1983 on the locally compact Ablian groups $G$. When $G=\mathbb{R}^{n}$, the modulation space can be equivalently defined by using the unit-cube decomposition to the frequency space (see Appendix A in [13], also [14, 17]). The following definition is based on the unit-cube decomposition introduced in [13].

Let $\sigma$ be a fixed nonnegative-valued function in $\mathcal{S}\left(\mathbb{R}^{n}\right)$ with support in the cube $[-4 / 5,4 / 5]^{n}$ and satisfy $\sigma(\xi)=1$ for any $\xi$ in the cube $[-2 / 5,2 / 5]^{n}$. By a standard constructive method, we may assume that for all $\xi \in \mathbb{R}^{n}$,

$$
\sum_{k \in \mathbb{Z}^{n}} \sigma_{k}(\xi)=1
$$

where $\sigma_{k}$ is the $k$-shift of $\sigma$ that is defined by

$$
\sigma_{k}(\xi)=\sigma(\xi-k), \quad \sigma_{0}(\xi)=\sigma(\xi) .
$$

For each $k \in \mathbb{Z}^{n}$, we use $\sigma_{k}(\xi)$ as its symbol of a smooth projection $\pi_{k}$ on the frequency space. Precisely, for any $f \in$ $\mathcal{S}^{\prime}\left(\mathbb{R}^{n}\right)$, we have

$$
\widehat{\pi_{k} f}=\sigma_{k} \widehat{f} .
$$

Let $X$ be a Banach space of measurable functions on $\mathbb{R}^{n}$ with quasi-norm $\|\cdot\|_{X}$. We define the modulation space

$$
M^{s}\left(X, \ell^{q}\right)\left(\mathbb{R}^{n}\right)=\left\{f \text { is measurable }:\|f\|_{M^{s}\left(X, \ell^{q}\right)}<\infty\right\},
$$

where

$$
\begin{gathered}
\|f\|_{M^{s}\left(X, \ell^{q}\right)}=\left\|\langle k\rangle^{s}\right\| \pi_{k}(f)\left\|_{X}\right\|_{\ell^{q}}, \\
\langle k\rangle=1+|k| .
\end{gathered}
$$

By definition, we have the inclusion

$$
M^{s}\left(X, \ell^{q}\right) \subset M^{r}\left(X, \ell^{q}\right) \quad \text { if } r \leq s .
$$

It is known that the definition of the modulation space $M^{s}\left(X, \ell^{q}\right)$ is independent of the choice of functions $\sigma$. In this paper, we are particularly interested in the cases $X=L^{p}$ and $X=H^{p}$, where $L^{p}$ is the Lebesgue space and $H^{p}$ is the real Hardy space. For all $0<p, q \leq \infty$, we call $M^{s}\left(L^{p}, \ell^{q}\right)$ 
the modulation spaces and $M^{s}\left(H^{p}, \ell^{q}\right)$ the modulation Hardy space. As a usual notation

$$
M^{s}\left(L^{p}, \ell^{q}\right)=M_{p, q}^{s}=M_{p, q}^{s}\left(\mathbb{R}^{n}\right)
$$

we similarly define

$$
M^{s}\left(H^{p}, \ell^{q}\right)=\mu_{p, q}^{s}=\mu_{p, q}^{s}\left(\mathbb{R}^{n}\right)
$$

By the definition and known properties of $H^{p}$, we have that for all $1<p<\infty$,

$$
\mu_{p, q}^{s}\left(\mathbb{R}^{n}\right)=M_{p, q}^{s}\left(\mathbb{R}^{n}\right)
$$

and for all $0<p \leq 1$,

$$
\mu_{p, q}^{s}\left(\mathbb{R}^{n}\right) \subset M_{p, q}^{s}\left(\mathbb{R}^{n}\right)
$$

For simplicity in notation, we denote

$$
\mu_{p, q}\left(\mathbb{R}^{n}\right)=\mu_{p, q}^{0}\left(\mathbb{R}^{n}\right), \quad M_{p, q}\left(\mathbb{R}^{n}\right)=M_{p, q}^{0}\left(\mathbb{R}^{n}\right)
$$

The following imbedding relation can be found in Proposition 5.1 .5 of [18]. Let $s_{1}, s_{2} \in \mathbb{R}, 0<p_{1}, p_{2}, q_{1}, q_{2} \leq \infty$. If

$$
s_{2} \leq s_{1}, \quad p_{1} \leq p_{2}, \quad q_{1} \leq q_{2}
$$

then

$$
M_{p_{1}, q_{1}}^{s_{1}} \subset M_{p_{2}, q_{2}}^{s_{2}}
$$

2.2. $H^{p}$ Spaces. It is well known that the Hardy space $H^{p}\left(\mathbb{R}^{n}\right)$ coincides with the Lebesgue space $L^{p}\left(\mathbb{R}^{n}\right)$ when $1<p<\infty$. For $0<p \leq 1$, the space $H^{p}\left(\mathbb{R}^{n}\right)$ has many characterizations. We will use its Riesz transform characterization in this paper. For an integer $L \geq 0$ and multi-index $J=\left(j_{1}, \ldots, j_{N}\right) \epsilon$ $\{1,2, \ldots, n\}^{L}$, let $R_{J}$ denote the generalized Riesz transform

$$
R_{J}(f)=R_{j_{1}} R_{j_{2}} R_{j_{L}}(f),
$$

where each $R_{j}(f)$ is the $j$ th Riesz transform of $f$ if $j \neq 0$ and $R_{0}(f)=f$. It is known that for $p>(n-1) /(n-$ $1+L)$ and all $f \in H^{p} \cap L^{2}$,

$$
\|f\|_{H^{p}} \simeq \sum_{J}\left\|R_{J}(f)\right\|_{L^{p}}
$$

where $\sum_{J}$ is a sum of finite terms.

The operator $\pi_{k} e^{i t|\Delta|^{\alpha / 2}}$ is a convolution. We have

$$
\left\|\pi_{k} e^{i t|\Delta|^{\alpha / 2}} f\right\|_{H^{p}} \simeq \sum_{J}\left\|\pi_{k} e^{i t|\Delta|^{\alpha / 2}} R_{J}(f)\right\|_{L^{p}} .
$$

Also it is well known that $R_{J}$ is bounded on $H^{p}$ spaces for any $0<p<\infty$.

\subsection{Some Lemmas and Known Results}

Lemma 8. Let $0<p<\infty$ and $t \geq 1$. Suppose that there is an integer $N>0$, such that for all test functions $f$

$$
\left\|\pi_{k} e^{i t|\Delta|^{\alpha / 2}} f\right\|_{L^{p}} \preceq t^{b_{1}}\|f\|_{H^{p}}
$$

for $|k|<N$ and

$$
\left\|\pi_{k} e^{i t|\Delta|^{\alpha / 2}} f\right\|_{L^{p}} \preceq t^{b_{2}}|k|^{d}\|f\|_{H^{p}}
$$

for $|k| \geq N$. Here $b_{1} \geq b_{2} \geq 0$ and $d$ is a real number. Then for $f \in H^{p} \cap L^{2}$, one has

$$
\left\|e^{i t|\Delta|^{\alpha / 2}} f\right\|_{M_{p, q}^{s}} \preceq t^{b_{1}}\|f\|_{M_{p, q}^{s-v}}+t^{b_{2}}\|f\|_{M_{p, q}^{s+d}},
$$

where $v$ is an arbitrary positive number.

Proof. The case $p \geq 1$ is proved in [11]. It suffices to show the lemma for $0<p \leq 1$. By the Riesz transform characterization of $H^{p}$, for $|k| \geq N$, we have

$$
\begin{aligned}
\left\|\pi_{k} e^{i t|\Delta|^{\alpha / 2}} f\right\|_{H^{p}} & \simeq \sum_{J}\left\|\pi_{k} e^{i t|\Delta|^{\alpha / 2}} R_{J}(f)\right\|_{L^{p}} \\
& \preceq \sum_{J} t^{b_{2}}|k|^{d}\left\|R_{J} f\right\|_{L^{p}} \simeq t^{b_{2}}|k|^{d}\|f\|_{H^{p}} .
\end{aligned}
$$

By checking the Fourier transform, we have the identity

$$
\pi_{k}=\sum_{|j|_{\infty} \leq 1} \pi_{k+j} \pi_{k}
$$

where

$$
j=\left(j_{1}, \ldots, j_{n}\right), \quad|j|_{\infty}=\max \left\{\left|j_{1}\right|, \ldots,\left|j_{n}\right|\right\} .
$$

So for $|k| \geq N-1$, one has

$$
\begin{aligned}
& \left\|\pi_{k} e^{i t|\Delta|^{\alpha / 2}} f\right\|_{H^{p}} \\
& =\left\|\sum_{|j|_{\infty} \leq 1} \pi_{k+j} \pi_{k}\left(S_{\alpha}(t) f\right)\right\|_{H^{p}} \\
& \quad \leq \sum_{|j|_{\infty} \leq 1}\left\|\pi_{k+j} e^{i t|\Delta|^{\alpha / 2}}\left(\pi_{k} f\right)\right\|_{H^{p}} \preceq t^{b_{2}}|k|^{d}\left\|\pi_{k} f\right\|_{H^{p}} .
\end{aligned}
$$

A similar argument shows that for $|k|<N-1$,

$$
\left\|\pi_{k} e^{i t|\Delta|^{\alpha / 2}} f\right\|_{H^{p}} \preceq t^{b_{1}}\left\|\pi_{k} f\right\|_{H^{p}}
$$

for any $f \in H^{p} \cap L^{2}$. The rest of the lemma easily follows from the definition of the modulation spaces.

Lemma 9 (see $[18,19])$. Let $E \subset \mathbb{R}^{n}$ denote an open set and $\psi \in C_{0}^{\infty}(E)$. If $\phi \in C^{\infty}(E)$ and the rank of the matrix

$$
\left(D_{x_{j}} D_{x_{i}} \phi(x)\right)_{i, j=1}^{n}
$$

is at least $m>0$ for all $x \in \operatorname{supp}(\psi)$, then

$$
\left|\int_{\mathbb{R}^{n}} e^{i \lambda \phi(x)} \psi(x) d x\right| \preceq|\lambda|^{-m / 2}\|\psi\|_{C^{2 n}} .
$$


Lemma 10. Let $2 \geq \alpha>0$ and $\alpha \neq 1$. Suppose that $\widetilde{\sigma}$ is a $C^{\infty}$ function with support in $[-1,1]^{n}$. Then

$$
\left\|\mathscr{F}^{-1}\left(\widetilde{\sigma}(\xi) e^{i t|\xi|^{\alpha}}\right)\right\|_{L^{\infty}\left(\mathbb{R}^{n}\right)} \preceq \min \left\{1, t^{-n / 2}\right\} .
$$

Proof. The case $\alpha=2$ is known [20]. It then suffices to show that for $0<\alpha<2$,

$$
\left\|\mathscr{F}^{-1}\left(\widetilde{\sigma}(\xi) e^{i t|\xi|^{\alpha}}\right)\right\|_{L^{\infty}\left(\mathbb{R}^{n}\right)} \leq t^{-n / 2}
$$

for large $t$. Let $\Gamma$ be a standard bump radial function supported in the set

$$
\left\{\xi: \frac{1}{2}<|\xi| \leq 2\right\}
$$

and satisfying, for all $\xi \neq 0$,

$$
\sum_{j} \Gamma\left(2^{j}|\xi|\right)=1
$$

Noting the support condition of $\widetilde{\sigma}$, we write

$$
\begin{aligned}
& \left|\mathscr{F}^{-1}\left(\widetilde{\sigma}(\xi) e^{i t|\xi|^{\alpha}}\right)(x)\right| \\
& \quad \simeq\left|\int_{\mathbb{R}^{n}} \widetilde{\sigma}(\xi) e^{i t|\xi|^{\alpha}} e^{i(x \cdot \xi)} d \xi\right| \\
& \quad \simeq\left|\sum_{j=-\infty}^{\infty} \int_{\mathbb{R}^{n}} \Gamma\left(2^{j}|\xi|\right) \widetilde{\sigma}(\xi) e^{i t|\xi|^{\alpha}} e^{i(x \cdot \xi)} d \xi\right| \\
& \quad \leq\left|\sum_{j \in A_{1} \cup A_{2} \cup A_{3}}^{\infty} 2^{-j n} \int_{\mathbb{R}^{n}} \Gamma(|\xi|) \widetilde{\sigma}\left(2^{-j} \xi\right) e^{i t 2^{-j \alpha}|\xi|^{\alpha}} e^{i 2^{-j}(x \cdot \xi)} d \xi\right|,
\end{aligned}
$$

where the sets $A_{k}, k=1,2,3$ are defined by

$$
\begin{gathered}
A_{1}=\left\{j \geq 0: 2^{-j(\alpha-1)-5} \geq \frac{|x|}{\alpha t}\right\}, \\
A_{2}=\left\{j \geq 0: n 2^{-j(\alpha-1)+5} \leq \frac{|x|}{\alpha t}\right\}, \\
A_{3}=\left\{j \geq 0: \frac{2^{5}|x|}{\alpha t}>2^{j(1-\alpha)}>\frac{2^{-5}|x|}{\alpha t n}\right\} .
\end{gathered}
$$

For $j \in A_{1}$, we use polar coordinates to write

$$
\begin{array}{r}
2^{-j n} \int_{\mathbb{R}^{n}} \Gamma(|\xi|) \widetilde{\sigma}\left(2^{-j} \xi\right) e^{i t 2^{-j \alpha}|\xi|^{\alpha}} e^{i 2^{-j}(x \cdot \xi)} d \xi \\
=2^{-j n} \int_{S^{n-1}}\left\{\int_{0}^{\infty} r^{n-1} \Gamma(r) \widetilde{\sigma}\left(\frac{2^{-j} r \xi}{|\xi|}\right)\right. \\
\left.\times e^{i t 2^{-j \alpha} r^{\alpha}} e^{i 2^{-j} r(x \cdot(\xi / \xi \xi))} d r\right\} d \xi^{\prime},
\end{array}
$$

where $d \xi^{\prime}$ is the induced Lebesgue measure on the unit sphere $S^{n-1}$. When $n$ is even, taking integration by parts for $n / 2$ times on the inside integral, we obtain

$$
\begin{gathered}
\sum_{j \in A_{1}}\left|2^{-j n} \int_{\mathbb{R}^{n}} \Gamma(|\xi|) \widetilde{\sigma}\left(2^{-j} \xi\right) e^{i t 2^{-j \alpha}|\xi|^{\alpha}} e^{i 2^{-j}(x \cdot \xi)} d \xi\right| \\
\leq t^{-n / 2} \sum_{j \in A_{1}} 2^{-j n(1-\alpha / 2)} \preceq t^{-n / 2} .
\end{gathered}
$$

When $n$ is odd, we use integration by parts for $n / 2+1 / 2$ times on the inside integral,

$$
\begin{aligned}
& \left|\int_{\mathbb{R}^{n}} \Gamma(|\xi|) \widetilde{\sigma}\left(2^{-j} \xi\right) e^{i t 2^{-j \alpha}|\xi|^{\alpha}} e^{i 2^{-j}(x \cdot \xi)} d \xi\right| \\
& \quad \leq\left|\int_{\mathbb{R}^{n}} \Gamma(|\xi|) \widetilde{\sigma}\left(2^{-j} \xi\right) e^{i t 2^{-j \alpha}|\xi|^{\alpha}} e^{i 2^{-j}(x \cdot \xi)} d \xi\right|^{n /(n+1)} \\
& \quad \leq t^{-n / 2} 2^{j \alpha n / 2} .
\end{aligned}
$$

Again we obtain that for odd $n$,

$$
\sum_{j \in A_{1}}\left|2^{-j n} \int_{\mathbb{R}^{n}} \Gamma(|\xi|) \widetilde{\sigma}\left(2^{-j} \xi\right) e^{i t 2^{-j \alpha}|\xi|^{\alpha}} e^{i 2^{-j}(x \cdot \xi)} d \xi\right| \preceq t^{-n / 2} .
$$

For $j \in A_{2}$, without loss of generality, we assume $\left|x_{1}\right| \geq$ $(|x| / n)$. Perform integration by parts on the $\xi_{1}$ variable for suitable amount of times. We similarly obtain

$$
\sum_{j \in A_{2}}\left|2^{-j n} \int_{\mathbb{R}^{n}} \Gamma(|\xi|) \widetilde{\sigma}\left(2^{-j} \xi\right) e^{i t 2^{-j \alpha}|\xi|^{\alpha}} e^{i 2^{-j}(x \cdot \xi)} d \xi\right| \preceq t^{-n / 2} .
$$

For $j \in A_{3}$, invoking Lemma 9, we obtain

$$
\begin{aligned}
& \left|\int_{\mathbb{R}^{n}} \Gamma(|\xi|) \widetilde{\sigma}\left(2^{-j} \xi\right) e^{i\left(t 2^{-j \alpha}|\xi|^{\alpha}+2^{-j}(x \cdot \xi)\right)} d \xi\right| \\
& \quad \preceq \min \left\{1, \frac{2^{j \alpha n / 2}}{t^{n / 2}}\right\} .
\end{aligned}
$$

Noting that $A_{3}$ contains no more than $(10 /|1-\alpha|)+\log _{2} n$ numbers of $j$, it is easy to check

$$
\sum_{j \in A_{3}} 2^{-j n} \min \left\{1, \frac{2^{j \alpha n / 2}}{t^{n / 2}}\right\} \leq t^{-n / 2} .
$$

The lemma is proved.

Lemma 11 (see [21, pages 163-171]). Let $0<p<2$ and

$$
N=\left[n\left(\frac{1}{p}-\frac{1}{2}\right)\right]+1 .
$$

Suppose that $T_{m}$ is a Fourier multiplier with symbol $m$. If $m$ is a bounded function which is of class $C^{N}$ in $\mathbb{R}^{n} \backslash\{0\}$ and if

$$
\left|\left(\frac{\partial}{\partial \xi}\right)^{J} m(\xi)\right| \leq\left(A|\xi|^{-1}\right)^{|J|} \text { for }|J| \leq N
$$

with $A \geq 1$, then $T_{m}$ is a bounded operator on $H^{p}$ and

$$
\left\|T_{m}\right\|_{H^{p} \rightarrow H^{p}} \preceq A^{n(1 / p-1 / 2)} .
$$


Lemma 12. Let $|k| \geq 1$ and $\alpha \neq 1$. For all $1 \leq p \leq \infty$, one has $\left\|\pi_{k} e^{i t|\Delta|^{\alpha / 2}} f\right\|_{L^{p}} \preceq\left\|\pi_{k} f\right\|_{L^{p}} \quad$ if $t|k|^{\alpha-2} \leq 1$,

$\left\|\pi_{k} e^{i t|\Delta|^{\alpha / 2}} f\right\|_{L^{p}}$

$$
\preceq\left(t|k|^{\alpha-2}\right)^{n|(1 / p)-(1 / 2)|}\left\|\pi_{k} f\right\|_{L^{p}} \quad \text { if } t|k|^{\alpha-2} \geq 1 .
$$

This lemma can be found in Section 4.2 of [11].

Lemma 13. Let $F$ be a compact subset in $\mathbb{R}^{n}$, and let $0<$ $p \leq q \leq \infty$. There exists a constant $C$ depending only on the diameter of $F$ and $p$, such that

$$
\|f\|_{L^{q}} \leq C\|f\|_{L^{p}}
$$

for all $f \in L^{p}$ satisfying supp $\hat{f} \subset F$.

This lemma is the Nikol'skij-Triebel inequality, see Proposition 1.3.2 in [20] (also Lemma 2.5 in [22]).

Lemma 14. Let $0<p \leq 1$ and $F, F^{\prime}$ be compact subsets of $\mathbb{R}^{n}$. Then there exists a constant $C$ depending only on the diameters of $F, F^{\prime}$ and $p$, such that

$$
\||f| *|g|\|_{L^{p}} \leq C\|f\|_{L^{p}}\|g\|_{L^{p}}
$$

for all $f, g \in L^{p}$ satisfying supp $\hat{f} \subset F$ and supp $\hat{g} \subset F^{\prime}$. [20]).

This is Lemma 2.6 in [22] (see also Proposition 1.5.3 in

Lemma 15 (Pitt's theorem). If $1<p \leq 2$ and $0 \leq \alpha<1 / p^{\prime}$, then

$$
\left(\int_{\mathbb{R}^{n}}|\widehat{f}(x)|^{p^{\prime}}|x|^{-\alpha n p^{\prime}} d x\right)^{1 / p^{\prime}} \preceq\left(\int_{\mathbb{R}^{n}}|f(x)|^{p}|x|^{\alpha n p} d x\right)^{1 / p} .
$$

Lemma 16. Let $s \in \mathbb{R}$ and $p, p_{j} \geq 1(j=1,2, \ldots, m)$ satisfy

$$
\frac{1}{p}=\frac{1}{p_{1}}+\frac{1}{p_{2}}+\cdots+\frac{1}{p_{m}}
$$

Then one has

$$
\left\|\prod_{j=1}^{m} u_{j}\right\|_{M_{p, 1}^{s}} \preceq \prod_{j=1}^{m}\left\|u_{j}\right\|_{M_{p_{j}, 1}^{s}} .
$$

This result is a particular case of Lemma 2.5 in [8].

\section{Proof of Theorem 1}

The operator $\pi_{k} e^{i t|\Delta|^{\alpha / 2}}$ is a convolution operator with the symbol $\sigma(\xi-k) e^{i t|\xi|^{\alpha}}$. This symbol is a $C^{\infty}$ function on $\mathbb{R}^{n} \backslash\{0\}$ with compact support. Clearly for any $N>0$ and $\xi \neq 0$, we have that for $|k| \leq 10$,

$$
\left|\left(\frac{\partial}{\partial \xi}\right)^{J} \sigma(\xi-k) e^{i t|\xi|^{\alpha}}\right| \preceq\left\{(1+t)|\xi|^{-1}\right\}^{|J|} \text { for }|J| \leq N .
$$

So Lemma 11 implies the following estimate.
Proposition 17. Let $0<p<\infty$. For any $k$ with $|k| \leq 10$, one has

$$
\left\|\pi_{k} e^{i t|\Delta|^{\alpha / 2}} f\right\|_{H^{p}} \preceq \max \left\{1, t^{n|1 / p-1 / 2|}\right\}\|f\|_{H^{p}} .
$$

By the proof of Lemma 8 and Proposition 17, we have that for all $|k| \leq 9$,

$$
\left\|\pi_{k} e^{i t|\Delta|^{\alpha / 2}} f\right\|_{H^{p}} \preceq \max \left\{1, t^{n|1 / p-1 / 2|}\right\}\left\|\pi_{k} f\right\|_{H^{p}} .
$$
$(0,1]$.

The following proposition extends Lemma 12 to all $p \in$

Proposition 18. Let $0<p \leq 1$. For any $k$ with $|k| \geq 1$, for any $\alpha \in \mathbb{R} \backslash\{0,1\}$, one has

$$
\begin{aligned}
& \left\|\pi_{k} e^{i t|\Delta|^{\alpha / 2}} f\right\|_{L^{p}} \\
& \quad \preceq \max \left\{1,\left(t|k|^{\alpha-2}\right)^{n(1 / p-1 / 2)}\right\} \sum_{|j|_{\infty} \leq 1}\left\|\pi_{k+j} f\right\|_{L^{p}} .
\end{aligned}
$$

Proof. The proof uses the same idea used in proving the case $p \geq 1$ which was represented in [11]. For the convenience of the reader, we present its proof.

Let $\Omega_{k}$ be the kernel of $\pi_{k} e^{i t|\Delta|^{\alpha / 2}}$. Then

$$
\Omega_{k}=\mathscr{F}^{-1}\left(\sigma(\xi-k) e^{i t|\xi|^{\alpha}}\right) .
$$

By Lemma 14 and (46), we have

$$
\left\|\pi_{k} e^{i t|\Delta|^{\alpha / 2}} f\right\|_{L^{p}} \preceq\left\|\Omega_{k}\right\|_{L^{p}} \sum_{|j|_{\infty} \leq 1}\left\|\pi_{k+j} f\right\|_{L^{p}} .
$$

Thus to prove the proposition, it suffices to show

$$
\left\|\mathscr{F}^{-1}\left(\sigma(\xi-k) e^{i t|\xi|^{\alpha}}\right)\right\|_{L^{p}} \preceq \max \left\{1,\left(t|k|^{\alpha-2}\right)^{n(1 / p-1 / 2)}\right\} .
$$

For simplicity, we prove the case $n=2$. The proof for $n \geq$ 3 , is tedious but shares the same idea as that for $n=2$.

First we study the case $t|k|^{\alpha-2}>1$. For $i=1,2$, and $k=$ $\left(k_{1}, k_{2}\right)$, if $\alpha \geq 2$ we denote

$$
\begin{aligned}
& C_{i}(k)=\alpha t\left(\left|k_{i}\right|+1\right)\left\{\left(\left|k_{1}\right|+1\right)^{2}+\left(\left|k_{2}\right|+1\right)^{2}\right\}^{(\alpha / 2)-1}, \\
& D_{i}(k)=\alpha t\left(\left|k_{i}\right|-1\right)\left\{\left(\left|k_{1}\right|-1\right)^{2}+\left(\left|k_{2}\right|-1\right)^{2}\right\}^{(\alpha / 2)-1} .
\end{aligned}
$$

If $\alpha<2$, we denote

$$
\begin{aligned}
& C_{i}(k)=|\alpha| t\left(\left|k_{i}\right|+1\right)\left\{\left(\left|k_{1}\right|-1\right)^{2}+\left(\left|k_{2}\right|-1\right)^{2}\right\}^{(\alpha / 2)-1}, \\
& D_{i}(k)=|\alpha| t\left(\left|k_{i}\right|-1\right)\left\{\left(\left|k_{1}\right|+1\right)^{2}+\left(\left|k_{2}\right|+1\right)^{2}\right\}^{(\alpha / 2)-1} .
\end{aligned}
$$


Also, for $i=1,2$ and $j \in \mathbb{N}$, we define sets

$$
\begin{gathered}
F_{i}=\left\{x_{i} \in \mathbb{R}: D_{i}(k)-t|k|^{\alpha-2}<\left|x_{i}\right| \leq C_{i}(k)+t|k|^{\alpha-2}\right\}, \\
G_{i, j}=\left\{x_{i} \in \mathbb{R}: C_{i}(k)+t|k|^{\alpha-2}+j-1\right. \\
\left.<\left|x_{i}\right| \leq C_{i}(k)+t|k|^{\alpha-2}+j\right\}, \\
H_{i, j}=\left\{x_{i} \in \mathbb{R}: D_{i}(k)-t|k|^{\alpha-2}-j\right. \\
\left.<\left|x_{i}\right| \leq D_{i}(k)-t|k|^{\alpha-2}-j+1\right\} .
\end{gathered}
$$

It is easy to check

$$
\begin{gathered}
\text { Length }\left(F_{i}\right) \preceq t|k|^{\alpha-2}, \\
\text { Length }\left(G_{i, j}\right)=\operatorname{Length}\left(H_{i, j}\right)=1 .
\end{gathered}
$$

Let

$$
K_{i, j}=G_{i, j} \cup H_{i, j}
$$

We have for $i=1,2$,

$$
\chi_{F_{i}}\left(x_{i}\right)+\sum_{j=1}^{\infty} \chi_{K_{i, j}}\left(x_{i}\right) \equiv 1
$$

Write

$$
\begin{aligned}
\int_{\mathbb{R}^{2}}\left|\mathscr{F}^{-1}\left(\sigma_{k}(\xi) e^{i t|\xi|^{\alpha}}\right)(x)\right|^{p} d x \\
=\int_{\mathbb{R}^{2}}\left|\mathscr{F}^{-1}\left(\sigma(\xi) e^{i t|\xi+k|^{\alpha}}\right)(x)\right|^{p} d x \\
\leq \int_{\mathbb{R}^{2}} \chi_{F_{1}}\left(x_{1}\right) \chi_{F_{2}}\left(x_{2}\right)\left|\mathscr{F}^{-1}\left(\sigma(\xi) e^{i t|\xi+k|^{\alpha}}\right)(x)\right|^{p} d x \\
+\sum_{j=1}^{\infty} \int_{\mathbb{R}^{2}} \chi_{K_{1, j}}\left(x_{1}\right) \chi_{F_{2}}\left(x_{2}\right) \\
\quad+\sum_{m=1}^{\infty} \int_{\mathbb{R}^{2}} \chi_{K_{2, m}}\left(x_{2}\right) \chi_{F_{1}}\left(x_{1}\right) \\
\quad \times\left|\mathscr{F}^{-1}\left(\sigma(\xi) e^{i t|\xi+k|^{\alpha}}\right)(x)\right|^{p} d x \\
+\sum_{j=1}^{\infty} \sum_{m=1}^{\infty} \int_{\mathbb{R}^{2}} \chi_{K_{1, j}}\left(x_{1}\right) \chi_{K_{2, m}}\left(x_{2}\right) \\
\times \mid I_{1}+I I_{2}+I I_{3}+I I_{4}, \\
\times\left|\mathscr{F}^{-1}\left(\sigma(\xi) e^{i t|\xi+k|^{\alpha}}\right)(x)\right|^{p} d x
\end{aligned}
$$

where

$$
\mathscr{F}^{-1}\left(\sigma(\xi) e^{i t|\xi+k|^{\alpha}}\right)(x) \simeq \int_{\mathbb{R}^{2}} e^{i\left(t|\xi+k|^{\alpha}+\langle x, \xi\rangle\right)} \sigma(\xi) d \xi .
$$

It is easy to check that if $t|k|^{\alpha-2} \geq 1$ and $\xi \in \operatorname{supp}(\sigma)$, the phase function

$$
\Phi(t, k, x, \xi)=t|\xi+k|^{\alpha}+(x \cdot \xi)
$$

satisfies

$$
\left|\operatorname{det} D_{\xi_{j}} D_{\xi_{i}} \Phi\right| \geq t|k|^{\alpha-2}
$$

So by Lemma 9, we have

$$
\begin{aligned}
I I_{1} & \leq\left(t^{-1}|k|^{2-\alpha}\right)^{p} \int_{\mathbb{R}^{2}} \chi_{F_{1}}\left(x_{1}\right) \chi_{F_{2}}\left(x_{2}\right) d x \\
& \leq\left(t^{-1}|k|^{2-\alpha}\right)^{p}\left(t|k|^{\alpha-2}\right)^{2}=t^{2-p}|k|^{(\alpha-2)(2-p)} .
\end{aligned}
$$

Observe the easy fact that if $x \in K_{1, j}$ and $\xi \in \operatorname{supp}(\sigma)$, for any integer $L$,

$$
\frac{\partial^{L}}{\partial \xi_{l}^{L}}\left(\frac{\sigma(\xi)}{\Phi_{\xi_{i}}(t, k, x, \xi)}\right)=O\left(\frac{1}{j+t|k|^{\alpha-2}}\right)^{L}, \quad i, l=1,2
$$

Perform integration by parts on $\xi_{1}$ and $\xi_{2}$ variables both for $L$ times such that $L p>1$. An easy computation shows that

$$
\begin{aligned}
I I_{4} \preceq & \sum_{j=1}^{\infty} \sum_{m=1}^{\infty} \int_{\mathbb{R}^{2}} \chi_{K_{1, j}}\left(x_{1}\right) \chi_{K_{2, m}}\left(x_{2}\right) \\
& \times \frac{1}{\left(j+t|k|^{\alpha-2}\right)^{L p}\left(m+t|k|^{\alpha-2}\right)^{L p}} \\
\preceq & \sum_{j \geq t|k|^{\alpha-2}}^{\infty} \sum_{m \geq t|k|^{\alpha-2}}^{\infty} \frac{1}{j^{L p} m^{L p}} \\
& \times \int_{\mathbb{R}^{2}} \chi_{K_{1, j}}\left(x_{1}\right) \chi_{K_{2, m}}\left(x_{2}\right) d x \preceq 1 .
\end{aligned}
$$

The estimates for $I_{2}$ and $I_{3}$ are exactly the same. We only estimate $I_{2}$. Take integration by parts on $\xi_{1}$ variable for $L$ times with $p L>1$. Again, a simple computation shows that

$$
\begin{aligned}
I I_{2} & \preceq \sum_{j \geq t|k|^{\alpha-2}}^{\infty} \frac{1}{j^{L p}} \int_{\mathbb{R}^{2}} \chi_{K_{1, j}}\left(x_{1}\right) \chi_{F_{2}}\left(x_{2}\right) d x \\
& \preceq\left(t|k|^{\alpha-2}\right)^{-L p+2} \preceq 1,
\end{aligned}
$$

if we chose a suitably large $L$. These estimates on $I I_{j}, j=$ $1,2,3,4$, indicate

$$
\begin{aligned}
\left\|\mathscr{F}^{-1}\left(\sigma(\xi-k) e^{i t|\xi|^{\alpha}}\right)\right\|_{L^{p}} & \leq t^{2 / p-1}|k|^{(\alpha-2)(2 / p-1)} \\
& =\left(t|k|^{\alpha-2}\right)^{2(1 / p-1 / 2)},
\end{aligned}
$$

provided $t|k|^{\alpha-2} \geq 1$ 
We now turn to show the case $t|k|^{\alpha-2}<1$. For $i=1,2$, and $k=\left(k_{1}, k_{2}\right)$, let $C_{i}(k) D_{i}(k)$ be the numbers defined above. For $i=1,2$ and $j \in \mathbb{N}$, we define sets

$$
\begin{gathered}
\mathfrak{F}_{i}=\left\{x_{i}: D_{i}(k)-1<\left|x_{i}\right| \leq C_{i}(k)+1\right\}, \\
\mathfrak{G}_{i, j}=\left\{x_{i}: C_{i}(k)+j<\left|x_{i}\right| \leq C_{i}(k)+1+j\right\}, \\
\mathfrak{H}_{i, j}=\left\{x_{i}: D_{i}(k)-1-j<\left|x_{i}\right| \leq D_{i}(k)-j\right\} .
\end{gathered}
$$

It is easy to check

$$
\begin{gathered}
\text { Length }\left(\mathfrak{F}_{i}\right) \preceq 1 \\
\operatorname{Length}\left(\mathfrak{G}_{i, j}\right)=\operatorname{Length}\left(\mathfrak{H}_{i, j}\right)=1 .
\end{gathered}
$$

Let

$$
\mathfrak{\Re}_{i, j}=\mathfrak{G}_{i, j} \cup \mathfrak{S}_{i, j} .
$$

Thus,

$$
\begin{aligned}
& \int_{\mathbb{R}^{2}}\left|\mathscr{F}^{-1}\left(\sigma_{k}(\xi) e^{i t|\xi|^{\alpha}}\right)(x)\right|^{p} d x \\
& \leq \int_{\mathbb{R}^{2}} \chi_{\mathfrak{F}_{1}}\left(x_{1}\right) \chi_{\mathfrak{F}_{2}}\left(x_{2}\right)\left|\mathscr{F}^{-1}\left(\sigma(\xi) e^{i t|\xi+k|^{\alpha}}\right)(x)\right|^{p} d x \\
& +\sum_{j=1}^{\infty} \int_{\mathbb{R}^{2}} \chi_{\mathfrak{\Re}_{1, j}}\left(x_{1}\right) \chi_{\mathfrak{F}_{2}}\left(x_{2}\right) \\
& \quad \times\left|\mathscr{F}^{-1}\left(\sigma(\xi) e^{i t|\xi+k|^{\alpha}}\right)(x)\right|^{p} d x \\
& \quad \sum_{m=1}^{\infty} \int_{\mathbb{R}^{2}} \chi_{\mathfrak{\Re}_{2, m}}\left(x_{2}\right) \chi_{\mathfrak{F}_{1}}\left(x_{1}\right) \\
& \quad \times\left|\mathscr{F}^{-1}\left(\sigma(\xi) e^{i t|\xi+k|^{\alpha}}\right)(x)\right|^{p} d x \\
& +\sum_{j=1}^{\infty} \sum_{m=1}^{\infty} \int_{\mathbb{R}^{2}} \chi_{\mathfrak{\Re}_{1, j}}\left(x_{1}\right) \chi_{\mathfrak{\Re}_{2, m}}\left(x_{2}\right) \\
& \times\left|\mathscr{F}^{-1}\left(\sigma(\xi) e^{i t|\xi+k|^{\alpha}}\right)(x)\right|^{p} d x .
\end{aligned}
$$

Using the same argument as we used before, we can show

$$
\left\|\mathscr{F}^{-1}\left(\sigma(\xi-k) e^{i t|\xi|^{\alpha}}\right)\right\|_{L^{p}} \preceq 1 .
$$

We complete the proof of Proposition 18.

We are now in a position to prove Theorem 1.

Proof. By an argument involving interpolation and duality, it suffices to show the case $p \leq 1$. Using Proposition 18, the inequality in (76) and the definition of the modulation spaces, we easily obtain (ii) in Theorem 1.

To show (i) and (iii) in Theorem 1, by Proposition 18 and the definition of the modulation spaces, it suffices to show

$$
\left\|\pi_{0} S_{\alpha}(t) f\right\|_{L^{p}} \preceq \max \left\{1, t^{n(1 / p-1 / 2)}\right\} \sum_{|j|_{\infty} \leq 1}\left\|\pi_{j} f\right\|_{L^{p}} .
$$

Again, by Lemma 14, the proof of the inequality in (101) can be reduced to show that for $t \geq 1$,

$$
\left(\int_{\mathbb{R}^{n}}\left|\int_{\mathbb{R}^{n}} \sigma(\xi) e^{i t|\xi|^{\alpha}} e^{i(\xi \cdot x)} d \xi\right|^{p} d x\right)^{1 / p} \preceq t^{n(1 / p-1 / 2)} .
$$

We show (iii) first. The proof of $n=1$ may illustrate the method. When $n=1$

$$
\begin{aligned}
& \left(\int_{\mathbb{R}}\left|\int_{\mathbb{R}} \sigma(\xi) e^{i t|\xi|^{\alpha}} e^{i(\xi \cdot x)} d \xi\right|^{p} d x\right)^{1 / p} \\
& \quad \leq\left(\int_{|x|<t}\left|\int_{\mathbb{R}} \sigma(\xi) e^{i t|\xi|^{\alpha}} e^{i(\xi \cdot x)} d \xi\right|^{p} d x\right)^{1 / p} \\
& \quad+\left(\int_{|x| \geq t}\left|\int_{\mathbb{R}} \sigma(\xi) e^{i t|\xi|^{\alpha}} e^{i(\xi \cdot x)} d \xi\right|^{p} d x\right)^{1 / p} .
\end{aligned}
$$

By Hölder's inequality and the Plancherel theorem, the first term above

$$
\begin{aligned}
& \left(\int_{|x|<t}\left|\int_{\mathbb{R}} \sigma(\xi) e^{i t|\xi|^{\alpha}} e^{i(\xi \cdot x)} d \xi\right|^{p} d x\right)^{1 / p} \\
& \quad \preceq\left(\int_{\mathbb{R}}\left|\sigma(\xi) e^{i t|\xi|^{\alpha}}\right|^{2} d \xi\right)^{1 / 2} t^{(1 / p-1 / 2)} \preceq t^{(1 / p-1 / 2)} .
\end{aligned}
$$

For the second term, performing integration by parts, we obtain

$$
\begin{aligned}
& \left(\int_{|x| \geq t}\left|\int_{\mathbb{R}} \sigma(\xi) e^{i t|\xi|^{\alpha}} e^{i(\xi \cdot x)} d \xi\right|^{p} d x\right)^{1 / p} \\
& =2\left(\int_{|x| \geq t}\left|\int_{0}^{\infty} \sigma(\xi) e^{i t \xi^{\alpha}} \cos \xi x d \xi\right|^{p} d x\right)^{1 / p} \\
& =2\left(\int_{|x|>t} \frac{1}{|x|^{p}} \mid \int_{0}^{+\infty}\left\{i \alpha t \sigma(\xi) \xi^{\alpha-1}+\sigma^{\prime}(\xi)\right\} e^{i t|\xi|^{\alpha}}\right. \\
& \left.\leq\left. 2\left(\int_{|x|>t} \frac{1}{|x|^{2 p /(2-p)}} d x\right)^{(2-p) / 2 p} \sin \xi x d \xi\right|^{p} d x\right)^{1 / p} \\
& \quad \times\left(\int_{|x|>t} \mid \int_{0}^{+\infty}\left\{i \alpha t \sigma(\xi) \xi^{\alpha-1}+\sigma^{\prime}(\xi)\right\}\right. \\
& \leq t\left(\int_{|x|>t} \frac{1}{\left.|x|^{2 p /(2-p)} d x\right)^{(2-p) / 2 p}}\right. \\
& \quad \times\left(\int_{R^{1}}\left(|\sigma(\xi)||\xi|^{\alpha-1}+\left|\sigma^{\prime}(\xi)\right|\right)^{2} d \xi\right)^{1 / 2} \preceq t^{1 / p-1 / 2} \\
& \left.\quad \times\left. e^{i t|\xi|^{\alpha}} \sin \xi x d \xi\right|^{2} d x\right)^{1 / 2}
\end{aligned}
$$

since

$$
\frac{2 p}{(2-p)}>1
$$


Now we return to show (i) of Theorem 1. We will prove only the case $\alpha<1$. Write

$$
\begin{gathered}
\left(\int_{\mathbb{R}^{n}}\left|\int_{\mathbb{R}^{n}} \sigma(\xi) e^{i t|\xi|^{\alpha}} e^{i(\xi \cdot x)} d \xi\right|^{p} d x\right)^{1 / p} \\
\preceq\left(\int_{|x|<t}\left|\int_{\mathbb{R}^{n}} \sigma(\xi) e^{i t|\xi|^{\alpha}} e^{i(\xi \cdot x)} d \xi\right|^{p} d x\right)^{1 / p} \\
\left(\int_{|x| \geq t}\left|\int_{\mathbb{R}^{n}} \sigma(\xi) e^{i t|\xi|^{\alpha}} e^{i(\xi \cdot x)} d \xi\right|^{p} d x\right)^{1 / p}=J_{1}+J_{2} .
\end{gathered}
$$

Using Hölder's inequality and the Plancherel theorem, we obtain

$$
J_{1} \preceq\left(\int_{\mathbb{R}^{n}}\left|\sigma(\xi) e^{i t|\xi|^{\alpha}}\right|^{2} d \xi\right)^{1 / 2} t^{n(1 / p-1 / 2)} \preceq t^{n(1 / p-1 / 2)} .
$$

For $i, j \in\{1,2, \ldots, n\}$, we denote sets

$$
\begin{gathered}
E_{t}=\left\{x \in \mathbb{R}^{n}:|x| \geq t\right\}, \\
E_{t, i}=\left\{x \in E_{t}:\left|x_{i}\right| \geq\left|x_{j}\right| \forall j \neq i\right\} .
\end{gathered}
$$

We now write

$$
J_{2} \preceq \sum_{i=1}^{n}\left(\int_{E_{t, i}}\left|\int_{\mathbb{R}^{n}} \sigma(\xi) e^{i t|\xi|^{\alpha}} e^{i(\xi \cdot x)} d \xi\right|^{p} d x\right)^{1 / p}
$$

To show (102), it now suffices to show that for each $i$,

$$
I=\left(\int_{E_{t, i}}\left|\int_{\mathbb{R}^{n}} \sigma(\xi) e^{i t|\xi|^{\alpha}} e^{i(\xi \cdot x)} d \xi\right|^{p} d x\right)^{1 / p} \preceq t^{n(1 / p-1 / 2)}
$$

Using the Leibniz rule, for any positive integer $L$, we have

$$
\partial_{i}^{(L)}\left(\sigma(x) e^{i t|x|^{\alpha}}\right)=\sum_{k=0}^{L} C_{L}^{k} \partial_{i}^{(L-k)}(\sigma(x)) \partial_{i}^{(k)}\left(e^{i t|x|^{\alpha}}\right)
$$

Here, an easy induction argument shows that, for $k \geq 1$,

$$
\partial_{i}^{(k)}\left(e^{i t|x|^{\alpha}}\right)=e^{i t|x|^{\alpha}} \sum_{j=1}^{k} t^{j} \varphi_{j \alpha-k}^{(k, j)}(x)
$$

where $\varphi_{j \alpha-k}^{(j . k)} \in C^{\infty}\left(R^{n}-\{0\}\right)$ is a homogeneous function of degree $j \alpha-k$ for each $j$. We now write

$$
\begin{aligned}
& \partial_{i}^{(L)}\left(\sigma(x) e^{i t|x|^{\alpha}}\right) \\
& \quad=e^{i t|x|^{\alpha}}\left(\partial_{i}^{(L)}(\sigma(x))+\sum_{j=1}^{L} t^{j}|x|^{j \alpha-L} \Phi_{j, L, \alpha}(x)\right),
\end{aligned}
$$

where

$$
\Phi_{j, L, \alpha}(x)=\sum_{k=j}^{L} C_{L}^{k} \partial_{i}^{(L-k)}(\sigma(x)) \varphi_{j \alpha-k}^{(k, j)}\left(\frac{x}{|x|}\right)|x|^{L-k} .
$$

By the definition, it is easy to see that each $\Phi_{j, L, \alpha}$ is an $L^{\infty}$ and $C^{\infty}\left(R^{n}-\{0\}\right)$ function with support in the cube $[-1 / 2,1 / 2]^{n}$.

Let $L=[(n / 2)+\alpha]+1$. Performing integration by parts on $\xi_{i}$ variables for $L$ times, we have

$$
\begin{aligned}
I= & \left(\int_{E_{t, i}}\left|\int_{R^{n}} \sigma(\xi) e^{i t|\xi|^{\alpha}} e^{i(x \cdot \xi)} d \xi\right|^{p} d x\right)^{1 / p} \\
& \leq\left(\int_{E_{t, i}}|x|^{-L p}\left|\int_{R^{n}} \partial_{i}^{(L)}(\sigma(\xi)) e^{i t|\xi|^{\alpha}} e^{i(x \cdot \xi)} d \xi\right|^{p} d x\right)^{1 / p} \\
& +\sum_{j=1}^{L} t^{j}\left(\left.\int_{E_{t, i}}|x|^{-L p}\left|\int_{R^{n}}\right| \xi\right|^{j \alpha-L} \Phi_{j, L, \alpha}\right. \\
\left.\times\left.(\xi) e^{i t|\xi|^{\alpha}} e^{i(x \cdot \xi)} d \xi\right|^{p} x\right)^{1 / p} & \\
= & \sum_{j=0}^{L} I_{j} .
\end{aligned}
$$

We first estimate each $I_{j}, 0 \leq j \leq L-1$. Recall that we assume $p \in(0,2)$. Let $q=2 / p$, so $p q^{\prime}=2 p /(2-p)$. By the choice of $L$ and the assumption

$$
p>\frac{n}{n+\alpha}
$$

it is easy to see $L p q^{\prime}>n$. Therefore, by Hölder's inequality, we obtain

$$
\begin{aligned}
I_{0} \leq & \left(\int_{|x| \geq t}|x|^{-L p q^{\prime}} d x\right)^{1 /\left(p q^{\prime}\right)} \\
& \times\left(\int_{R^{n}}\left|\int_{R^{n}} \partial_{i}^{(L)}(\sigma(\xi)) e^{i t|\xi|^{\alpha}} e^{i(\xi \cdot x)} d \xi\right|^{2} d x\right)^{1 / 2} \\
& \preceq t^{-L+n(1 / p-1 / 2)}\left\|\partial_{i}^{(L)} \sigma\right\|_{2} \preceq t^{-L+n(1 / p-1 / 2)} .
\end{aligned}
$$

For each $j=1,2, \ldots, L-1$, by the choice of $L$, the assumption on $p$, and an easy computation, it is not difficult to see that we may obtain a number $\alpha_{j}$ in the interval $[0, n / 2)$ satisfying

$$
\begin{gathered}
L-\alpha_{j}>n\left(\frac{1}{p}-\frac{1}{2}\right), \quad \alpha_{j}+j \alpha-L>-\frac{n}{2}, \\
\alpha_{j}+j-L \leq 0 .
\end{gathered}
$$


By Hölder's inequality and Pitt's theorem, for each $j$, we obtain

$$
\begin{aligned}
I_{j} \leq & t^{j}\left(\left.\int_{E_{t, i}}|x|^{-\left(L-\alpha_{j}\right) p}|| x\right|^{-\alpha_{j}} \int_{R^{n}}|\xi|^{j \alpha-L} \Phi_{j, L, \alpha}(\xi) e^{i t|\xi|^{\alpha}}\right. \\
\leq & \left.\times\left. e^{i(x \cdot \xi)} d \xi\right|^{p} d x\right)^{1 / p} \\
& \quad\left(\int_{|x| \geq t}|x|^{-\left(L-\alpha_{j}\right) p q^{\prime}} d x\right)^{1 /\left(p q^{\prime}\right)} \\
\leq & t^{-L+\alpha_{j}+j+n(1 / p-1 / 2)}\left(\left.\left.\int_{R^{n}}|| x\right|^{-\alpha_{j}} \int_{R^{n}}|\xi|^{j \alpha-L} \Phi_{j, L, \alpha}(\xi) e^{\alpha_{j}}|x|^{j \alpha-\left.\xi\right|^{\alpha}} e^{i(x \cdot \xi)} d \xi\right|^{2} d x\right)^{1 / 2} \\
\leq & t^{-L+\alpha_{j}+\alpha+n(1 / p-1 / 2)} .
\end{aligned}
$$

Combining all the estimates, we have

$$
\begin{gathered}
\left(\int_{E_{t, i}}\left|\int_{R^{n}} \sigma(\xi) e^{i t|\xi|^{\alpha}} e^{i(x \cdot \xi)} d \xi\right|^{p} d x\right)^{1 / p} \\
\leq I_{L}+\sum_{j=0}^{L-1} t^{-L+\alpha_{j}+j+n(1 / p-1 / 2)} \\
\leq I_{L}+t^{n(1 / p-1 / 2)} .
\end{gathered}
$$

It remains to estimate $I_{L}$.

It is easy to see that the choice of $L$ and the condition

$$
\alpha>\alpha_{0}= \begin{cases}\frac{2}{n+2} & \text { if } n \text { is even } \\ \frac{3}{n+3} & \text { if } n \text { is odd }\end{cases}
$$

in the theorem imply $L \alpha-L>-n / 2$. So, by Hölder's inequality and Pitt's theorem again, we obtain

$$
\begin{aligned}
I_{L}= & t^{L}\left(\left.\left.\int_{E_{t, i}}|x|^{-L p}\left|\int_{R^{n}}\right| \xi\right|^{L \alpha-L} \Phi_{L, L, \alpha}(\xi) e^{i t|\xi|^{\alpha}} e^{i(x \cdot \xi)} d \xi\right|^{p} d x\right)^{1 / p} \\
\leq & t^{L}\left(\int_{|x| \geq t}|x|^{-L p q^{\prime}} d x\right)^{1 /\left(p q^{\prime}\right)} \\
& \times\left(\left.\left.\int_{R^{n}}\left|\int_{R^{n}}\right| \xi\right|^{L \alpha-L} \Phi_{L, L, \alpha}(\xi) e^{i t|\xi|^{\alpha}} e^{i(x \cdot \xi)} d \xi\right|^{2} d x\right)^{1 / 2} \\
\leq & t^{n(1 / p-1 / 2)}\left\||\xi|^{L \alpha-L} \Phi_{L, L, \alpha}(\xi)\right\|_{2} \preceq t^{n(1 / p-1 / 2)} .
\end{aligned}
$$

This completes the proof of (102).
When $\alpha=2,4, \ldots$, we have

$$
\begin{aligned}
& \left(\int_{E_{t, i}}\left|\int_{\mathbb{R}^{n}} \sigma(\xi) e^{i t|\xi|^{\alpha}} e^{i(\xi \cdot x)} d \xi\right|^{p} d x\right)^{1 / p} \\
& \quad \leq t^{L}\left(\int_{E_{t, i}} \frac{1}{|x|^{p L}}\left|\int_{|\xi| \leq 2} \omega(\xi) e^{i t|\xi|^{\alpha}} e^{i(\xi \cdot x)} d \xi\right|^{p} d x\right)^{1 / p}
\end{aligned}
$$

for any integer $L$, where $\omega(\xi)$ is a $C^{\infty}$ function. Thus it is trivial to see that

$$
\left(\int_{E_{t, i}}\left|\int_{\mathbb{R}^{n}} \sigma(\xi) e^{i t|\xi|^{\alpha}} e^{i(\xi \cdot x)} d \xi\right|^{p} d x\right)^{1 / p} \leq t^{n(1 / p-1 / 2)}
$$

for all $p>0$. This proves (i) in Theorem 1 .

\section{Proof of Theorem 3}

Recall that the function $\sigma$ chosen in the definition of the modulation space is flexible. We may choose

$$
\sigma=\prod_{j=1}^{n} \theta_{j},
$$

where each $\theta_{j}$ is a nonnegative valued function in $S(\mathbb{R})$ with support in $[-4 / 5,4 / 5]$ and satisfies $\theta_{j}\left(\xi_{j}\right)=1$ if $\xi_{j} \epsilon$ $[-2 / 5,2 / 5]$ and

$$
\sum_{k_{j} \in \mathbb{Z}} \theta_{j, k_{j}}\left(\xi_{j}\right)=1, \quad \theta_{j, k_{j}}\left(\xi_{j}\right)=\theta_{j}\left(\xi_{j}+k_{j}\right) .
$$

For simplicity, we work on the case $0<p<2$. Let $\phi \in C^{\infty}(\mathbb{R})$ be a nonnegative function with support in the set $[1 / 16,1 / 8]$. For

$$
x=\left(x_{1}, x_{2}, \ldots, x_{n}\right),
$$

define a function $\Phi$ on $\mathbb{R}^{n}$ by

$$
\Phi(x)=\prod_{j=1}^{n} \phi\left(x_{j}\right) .
$$

and an $f \in S\left(\mathbb{R}^{n}\right)$ by $f=\mathscr{F}^{-1}(\Phi)$. Let $k \in \mathbb{Z}^{n}$. It is easy to see that

$$
\pi_{k}(f)(x)=\epsilon(k) f(x),
$$

where $\epsilon(k)=1$ if $k=0$ and $\epsilon(k)=0$ if $k \neq 0$. Similarly we have

$$
\pi_{k}\left(e^{i t|\Delta|} f\right)(x)=\epsilon(k)\left(e^{i t|\Delta|} f\right)(x) .
$$

Suppose that we have some $\delta$ such that

$$
\left\|e^{i t|\Delta|} f\right\|_{M_{p, q}^{s}\left(\mathbb{R}^{n}\right)} \leq t^{\delta}\|f\|_{M_{p, q}^{s}\left(\mathbb{R}^{n}\right)} .
$$

By the choice of $f$, we have

$$
\begin{aligned}
\left\|e^{i t|\Delta|} f\right\|_{M_{p, q}^{s}\left(\mathbb{R}^{n}\right)} & =\left\|\pi_{0} e^{i t|\Delta|} f\right\|_{L^{p}\left(\mathbb{R}^{n}\right)} \\
& \leq t^{\delta}\|f\|_{M_{p, q}^{s}\left(\mathbb{R}^{n}\right)}=t^{\delta}\|f\|_{L^{p}\left(\mathbb{R}^{n}\right)} .
\end{aligned}
$$


On the other hand,

$$
\left\|\pi_{0} e^{i t|\Delta|} f\right\|_{L^{p}\left(\mathbb{R}^{n}\right)}=\left(\int_{\mathbb{R}^{n}}\left|\prod_{j=1}^{n} \int_{\mathbb{R}} \phi\left(\xi_{j}\right) e^{i \psi\left(\xi_{j}, x_{j}\right)} d \xi_{j}\right|^{p} d x\right)^{1 / p},
$$

where the phase function $\psi\left(\xi_{j}, x_{j}\right)$ is defined by

$$
\psi\left(\xi_{j}, x_{j}\right)=t \xi_{j}^{2}+\xi_{j} x_{j}
$$

Since

$$
\frac{\partial}{\partial \xi_{j}} \psi\left(\xi_{j}, x_{j}\right)=2 t \xi_{j}+x_{j}
$$

The critical point of $\psi\left(\xi_{j}, x_{j}\right)$ is at

$$
\xi_{j}^{*}=\frac{-x_{j}}{2 t} .
$$

Thus, by the stationary phase method (see [19, Proposition 3 , page 334]), an easy computation gives that, as $t \rightarrow \infty$,

$$
\begin{aligned}
& \left(\int_{\mathbb{R}^{n}}\left|\prod_{j=1}^{n} \int_{\mathbb{R}} \phi\left(\xi_{j}\right) e^{i \psi\left(\xi_{j}, x_{j}\right)} d \xi_{j}\right|^{p} d x\right)^{1 / p} \\
& \geq\left(\prod_{j=1}^{n} \int_{\left|x_{j}\right| \leq 2 t}\left|\int_{\mathbb{R}} \phi\left(\xi_{j}\right) e^{i \psi\left(\xi_{j}, x_{j}\right)} d \xi_{j}\right|^{p} d x_{j}\right)^{1 / p} \\
& \simeq t^{-n / 2}\left(\prod_{j=1}^{n} \int_{\left|x_{j}\right| \leq 2 t}\left|\phi\left(\frac{-x_{j}}{2 t}\right)\right|^{p} d x_{j}\right)^{1 / p} \\
& \quad+O\left(t^{n(1 / p-1 / 2)-1 / 2}\right) \\
& \simeq t^{n(1 / p-1 / 2)}
\end{aligned}
$$

Thus the inequality

$$
\left\|e^{i t|\Delta|} f\right\|_{M_{p, q}^{s}\left(\mathbb{R}^{n}\right)} \preceq t^{\delta}\|f\|_{M_{p, q}^{s}\left(\mathbb{R}^{n}\right)}
$$

implies

$$
t^{n(1 / p-1 / 2)} \preceq t^{\delta}, \quad \text { as } t \longrightarrow \infty .
$$

This shows the conclusion.

\section{Applications}

5.1. Operators $S_{\alpha, \beta}(t)$. Let $\alpha \neq 1$ and $\beta \in \mathbb{R}$. In [23], to investigate the absolute convergence for multiple Fourier series, Wainger studied the oscillating multipliers $S_{\alpha, \beta}(\mathrm{t})$ with symbol

$$
m_{\alpha, \beta}(t, \xi)=\frac{e^{i t|\xi|^{\alpha}}}{\left(1+|\xi|^{2}\right)^{\beta / 2}}
$$

In [24], Miyachi proved that in the case $\alpha>0$ and $\alpha \neq 1$, for $0<p<\infty$,

$$
\left\|S_{\alpha, \beta}(1) f\right\|_{H^{p}\left(\mathbb{R}^{n}\right)} \preceq\|f\|_{H^{p}\left(\mathbb{R}^{n}\right)}
$$

if and only if

$$
\beta \geq n \alpha\left|\frac{1}{p}-\frac{1}{2}\right| .
$$

By Theorem 1 and its proof, we not only obtain the boundedness of $S_{\alpha, \beta}(t)$ on $\mu_{p, q}^{s}\left(\mathbb{R}^{n}\right)$ for any $\beta \in \mathbb{R}$, but also gain a regularity of $\beta$ if $\beta>0$.

Theorem 19. Let $0<p<\infty$ and $\alpha \neq 1$. One has for $t \geq 1$

$$
\left\|S_{\alpha, \beta}(t) f\right\|_{\mu_{p, q}^{s}} \preceq t^{n|1 / p-1 / 2|}\|f\|_{\mu_{p, q}^{s+\gamma-\beta}}+\|f\|_{\mu_{p, q}^{s},},
$$

where

$$
\gamma=n(\alpha-2)\left|\frac{1}{p}-\frac{1}{2}\right| .
$$

Particularly, if $\beta=n(\alpha-2)|1 / p-1 / 2|$, we have

$$
\left\|S_{\alpha, \beta}(t) f\right\|_{\mu_{p, q}^{s}} \preceq t^{n|1 / p-1 / 2|}\|f\|_{\mu_{p, q}^{s}} .
$$

Proof. The proof of Theorem 19 is the same as the proof of Theorem 1. We skip it.

5.2. $M_{p_{1}, q}^{s} \rightarrow M_{p_{2}, q}^{s}$ Boundedness. We will study the $M_{p_{1}, q}^{s} \rightarrow M_{p_{2}, q}^{s}$ boundedness for the operator $e^{i t|\Delta|^{\alpha / 2}}$ in the case $0<\alpha \leq 2$ and $\alpha \neq 1$. The other cases of $\alpha$ will be addressed in another paper. First we estimate $\pi_{k} e^{i t|\Delta|^{\alpha / 2}}$ for $|k| \leq 10$.

Proposition 20. Let $\alpha \neq 1,0<\alpha \leq 2$ and $|k| \leq 9$. One has for all $0<r \leq 1 \leq p \leq \infty$,

$$
\left\|\pi_{k} e^{i t|\Delta|^{\alpha / 2}} f\right\|_{L^{p}} \preceq(1+t)^{-n(1 / 2-1 / p)}\left\|\pi_{k}(f)\right\|_{L^{r}} .
$$

Proof. By Lemma 9, we know

$$
\left\|\pi_{k} e^{i t|\Delta|^{\alpha / 2}}\right\|_{L^{1} \rightarrow L^{\infty}} \leq(1+t)^{-n / 2} .
$$

On the other hand, it is known from [11]

$$
\left\|\pi_{k} e^{i||||^{\alpha / 2}}\right\|_{L^{p} \rightarrow L^{p}} \preceq(1+t)^{n|1 / p-1 / 2|}
$$

for all $1 \leq p \leq \infty$. Interpolating these two inequalities with the energy inequality

$$
\left\|\pi_{k} e^{i t|\Delta|^{\alpha / 2}}\right\|_{L^{2} \rightarrow L^{2}} \preceq 1,
$$

we find that for any $1 \leq p \leq \infty$,

$$
\left\|\pi_{k} e^{i t|\Delta|^{\alpha / 2}}\right\|_{L^{p} \rightarrow L^{\infty}} \leq(1+t)^{-n(1 / p-1 / 2)} .
$$


Let $1 \leq p \leq \infty$. For $f \in L^{1}$, we have

$$
\begin{aligned}
\left\|\pi_{k} e^{i t|\Delta|^{\alpha / 2}} f\right\|_{L^{p}} & =\sup _{\|g\|_{L^{p^{\prime}}}=1}\left|\left\langle\pi_{k} S_{\alpha}(t) f, g\right\rangle\right| \\
& =\sup _{\|g\|_{L^{p^{\prime}}}=1}\left|\left\langle f,\left(\pi_{k} e^{i t|\Delta|^{\alpha / 2}}\right)^{*} g\right\rangle\right| \\
& \preceq \sup _{\|g\|_{L^{p^{\prime}}}}\left\|\left(\pi_{k} e^{i t|\Delta|^{\alpha / 2}}\right)^{*} g\right\|_{L^{\infty}}\|f\|_{L^{1}} \\
& \preceq(1+t)^{n(1 / p-1 / 2)}\|f\|_{L^{1}} .
\end{aligned}
$$

This shows that for $|k| \leq 10$,

$$
\left\|\pi_{k} e^{i t|\Delta|^{\alpha / 2}} f\right\|_{L^{p}} \preceq(1+t)^{-n(1 / 2-1 / p)}\|f\|_{L^{1}} .
$$

Now if $0<r \leq 1$, we obtain that for all $1 \leq p \leq \infty$,

$$
\begin{aligned}
\left\|\pi_{k} e^{i t|\Delta|^{\alpha / 2}} f\right\|_{L^{p}} & \preceq \sum_{|\ell| \leq 1}\left\|\pi_{k+\ell} S_{\alpha}(t) \pi_{k}(f)\right\|_{L^{p}} \\
& \preceq(1+t)^{-n(1 / 2-1 / p)}\left\|\pi_{k}(f)\right\|_{L^{1}} \\
& \preceq(1+t)^{-n(1 / 2-1 / p)}\left\|\pi_{k}(f)\right\|_{L^{r}} .
\end{aligned}
$$

The last inequality is from Lemma 13.

As these discussions, we obtain the following corollary.

Corollary 21. Let $0<\alpha \leq 2, \alpha \neq 1,|k| \leq 10$ and $0<p_{1} \leq 1 \leq$ $p_{2} \leq \infty$, one has

$$
\begin{gathered}
\left\|\pi_{k} e^{i t|\Delta|^{\alpha / 2}} f\right\|_{H^{p_{2}}} \preceq\left\|\pi_{k} f\right\|_{H^{p_{1}}} \quad \text { if } t \leq 1, \\
\left\|\pi_{k} e^{i t|\Delta|^{\alpha / 2}} f\right\|_{H^{p_{2}}} \preceq t^{n\left(1 / p_{2}-1 / 2\right)}\left\|\pi_{k} f\right\|_{H^{p_{1}}} \quad \text { if } t>1,
\end{gathered}
$$

where for convenience, one denotes $L^{\infty}=H^{\infty}$.

Proposition 22. Let $0<\alpha \leq 2$ and $\alpha \neq 1$. One has for $|k| \geq 9$ and all $0<r \leq 1 \leq p \leq \infty$

$$
\left\|\pi_{k} e^{i t|\Delta|^{\alpha / 2}} f\right\|_{L^{p}} \preceq\left(1+t|k|^{\alpha-2}\right)^{-n(1 / 2-1 / p)}\left\|\pi_{k}(f)\right\|_{L^{r}} .
$$

Proof. By Lemma 9, it is trivial to see that

$$
\left\|\pi_{k} e^{i t|\Delta|^{\alpha / 2}}\right\|_{L^{1} \rightarrow L^{\infty}} \preceq \min \left\{1, t^{-n / 2}|k|^{-n(\alpha-2) / 2}\right\} .
$$

Interpolating this inequality with the energy inequality, we find that for any $p \leq 2$,

$$
\left\|\pi_{k} e^{i t|\Delta|^{\alpha / 2}}\right\|_{L^{p} \rightarrow L^{\infty}} \preceq\left(1+t|k|^{\alpha-2}\right)^{-n(1 / p-1 / 2)} .
$$

So by duality, for $|k| \geq 9$,

$$
\left\|\pi_{k} e^{i t|\Delta|^{\alpha / 2}} f\right\|_{L^{p}} \preceq\left(1+t|k|^{\alpha-2}\right)^{n(1 / p-1 / 2)}\|f\|_{L^{1}} .
$$

Now for any $0<r \leq 1$, we obtain that for all $1 \leq p \leq \infty$,

$$
\begin{aligned}
\left\|\pi_{k} e^{i t|\Delta|^{\alpha / 2}} f\right\|_{L^{p}} & \preceq \sum_{|\ell| \leq 1}\left\|\pi_{k+\ell} S_{\alpha}(t) \pi_{k}(f)\right\|_{L^{p}} \\
& \preceq\left(1+t|k|^{\alpha-2}\right)^{-n(1 / p-1 / 2)}\left\|\pi_{k}(f)\right\|_{L^{1}} \\
& \preceq\left(1+t|k|^{\alpha-2}\right)^{n(1 / p-1 / 2)}\left\|\pi_{k}(f)\right\|_{L^{r}}
\end{aligned}
$$

As a consequence the proposition, we have the following.

Corollary 23. Let $0<\alpha \leq 2, \alpha \neq 1,|k| \geq 9$ and $0<p_{1} \leq 1 \leq$ $p_{2} \leq \infty$, one has

$$
\begin{aligned}
& \left\|\pi_{k} e^{i t|\Delta|^{\alpha / 2}} f\right\|_{H^{p_{2}}} \preceq\left\|\pi_{k} f\right\|_{H^{p_{1}}} \quad \text { if } t|k|^{\alpha-2} \leq 1, \\
& \left\|\pi_{k} e^{i t|\Delta|^{\alpha / 2}} f\right\|_{H^{p_{2}}} \\
& \preceq|k|^{n(\alpha-2)\left(1 / p_{2}-1 / 2\right)} t^{n\left(1 / p_{2}-1 / 2\right)}\left\|\pi_{k} f\right\|_{H^{p_{1}}} \quad \text { if } t|k|^{\alpha-2} \geq 1 .
\end{aligned}
$$

By Corollaries 21 and 23 and the definition of the modulation spaces, we now obtain the $\mu_{p_{1}, q}^{s} \rightarrow M_{p_{2}, q}^{s}$ boundedness of $e^{i t|\Delta|^{\alpha / 2}}$ on the modulation spaces.

Theorem 24. Let $0<\alpha \leq 2, \alpha \neq 1$, and $0<p_{1} \leq 1 \leq p_{2} \leq \infty$, one has that

$$
\left\|e^{i t|\Delta|^{\alpha / 2}} f\right\|_{M_{p_{2}, q}^{s}} \preceq(1+t)^{n\left(1 / p_{2}-1 / 2\right)}\|f\|_{\mu_{p_{1}, q}^{s+\gamma}},
$$

where

$$
\gamma=n(\alpha-2)\left(\frac{1}{p_{2}}-\frac{1}{2}\right) .
$$

Next, we give the proof of Theorem 4 .

Proof. By the definition of the modulation space, we know

$$
\begin{aligned}
& \|\| e^{i t|\Delta|^{\alpha / 2}} f\left\|_{M_{p_{2}, q}^{s}\left(\mathbb{R}^{n}\right)}\right\|_{L^{r}(0, \infty)} \\
& =\left\{\int_{0}^{\infty}\left[\sum_{k}(1+|k|)^{s q}\left\|\pi_{k} e^{i t|\Delta|^{\alpha / 2}} f\right\|_{L^{p_{2}}}^{q}\right]^{r / q} d t\right\}^{1 / r} .
\end{aligned}
$$

If $r / q \geq 1$, write

$$
(1+|k|)^{s q}\left\|\pi_{k} S_{\alpha}(t) f\right\|_{L^{p_{2}}}^{q}=g_{k}(t) .
$$


By the Minkowski inequality, we have

$$
\begin{aligned}
\|\| e^{i t|\Delta|^{\alpha / 2}} f\left\|_{M_{p_{2}}^{s} q^{\prime}\left(\mathbb{R}^{n}\right)}\right\|_{L^{r}(0, \infty)} & \leq\left\{\sum_{k}\left(\int_{0}^{\infty}\left|g_{k}(t)\right|^{r / q} d t\right)^{q / r}\right\}^{1 / q} \\
& =\left\{\sum_{k}\left(\int_{0}^{\infty}(1+|k|)^{s r}\left\|\pi_{k} e^{i t|\Delta|^{\alpha / 2}} f\right\|_{L^{p_{2}}}^{r} d t\right)^{q / r}\right\}^{1 / q} \\
& =\left\{\sum_{k}(1+|k|)^{s q}\left(\int_{0}^{\infty}\left\|\pi_{k} e^{i t|\Delta|^{\alpha / 2}} f\right\|_{L^{p_{2}}}^{r} d t\right)^{q / r}\right\}^{1 / q} .
\end{aligned}
$$

When $|k| \leq 10$,

$$
\begin{aligned}
& \left(\int_{0}^{\infty}\left\|\pi_{k} e^{i t|\Delta|^{\alpha / 2}} f\right\|_{L^{p_{2}}}^{r} d t\right)^{q / r} \\
& \quad \preceq\left(\left\|\pi_{k} f\right\|_{H^{p_{1}}}^{r} \int_{0}^{\infty}(1+t)^{-r n\left(1 / 2-1 / p_{2}\right)} d t\right)^{q / r} \preceq\left\|\pi_{k} f\right\|_{H^{p_{1}}}^{q} .
\end{aligned}
$$

When $|k| \geq 9$,

$$
\begin{aligned}
\left(\int_{0}^{\infty}\left\|\pi_{k} e^{i t|\Delta|^{\alpha / 2}} f\right\|_{L^{p_{2}}}^{r} d t\right)^{q / r} & \leq\left(\int_{0}^{|k|^{2-\alpha}}\left\|\pi_{k} e^{i t|\Delta|^{\alpha / 2}} f\right\|_{L^{p_{2}}}^{r} d t\right)^{q / r} \\
& +\left(\int_{|k|^{2-\alpha}}^{\infty}\left\|\pi_{k} e^{i t|\Delta|^{\alpha / 2}} f\right\|_{L^{p_{2}}}^{r} d t\right)^{q / r} \\
& \leq\left(\left\|\pi_{k} f\right\|_{L^{p_{1}}}^{r} \int_{0}^{|k|^{2-\alpha}} d t\right)^{q / r} \\
& +\left\|\pi_{k} f\right\|_{L^{p_{1}}}^{q}\left(\int_{|k|^{2-\alpha}}^{\infty}\left(t|k|^{\alpha-2}\right)^{r n\left(1 / 2-1 / p_{2}\right)} d t\right)^{q / r} \\
\leq & \left\|\pi_{k} f\right\|_{L^{p_{1}}}^{q}|k|^{(2-\alpha) q / r} \\
& +\left\|\pi_{k} f\right\|_{L^{p_{1}}}^{q}\left(|k|^{(\alpha-2) r n\left(1 / p_{2}-1 / 2\right)} \int_{|k|^{2-\alpha}}^{\infty} t^{-r n\left(1 / 2-1 / p_{2}\right)} d t\right)^{q / r} \\
\leq & \left\|\pi_{k} f\right\|_{L^{p_{1}}}^{q}|k|^{(2-\alpha) q / r} .
\end{aligned}
$$

This shows that if $r \geq q$, then

$$
\begin{aligned}
& \|\| e^{i t|\Delta|^{\alpha / 2}} f\left\|_{M_{p_{2}, q}^{s}\left(\mathbb{R}^{n}\right)}\right\|_{L^{r}(0, \infty)} \\
& \preceq\left\{\sum_{k}(1+|k|)^{s q}\langle k\rangle^{(2-\alpha) q / r}\left\|\pi_{k} f\right\|_{L^{p_{1}}}^{q}\right\}^{1 / q} \\
& =\|f\|_{\mu_{p_{1}, q}^{s+(2-\alpha) / r}\left(\mathbb{R}^{n}\right)} .
\end{aligned}
$$

If $r / q \leq 1$, then

$$
\begin{aligned}
& \|\| e^{i t|\Delta|^{\alpha / 2}} f\left\|_{M_{p_{2}{ }^{\prime}}^{s}\left(\mathbb{R}^{n}\right)}\right\|_{L^{r}(0, \infty)} \\
& \quad \preceq\left\{\int_{0}^{\infty} \sum_{k}(1+|k|)^{s r}\left\|\pi_{k} e^{i t|\Delta|^{\alpha / 2}} f\right\|_{L^{p 2}}^{r} d t\right\}^{1 / r} .
\end{aligned}
$$

As in the previous case,

$$
\int_{0}^{\infty}\left\|\pi_{k} e^{i t|\Delta|^{\alpha / 2}} f\right\|_{L^{p_{2}}}^{r} d t \preceq\left\|\pi_{k} f\right\|_{H^{p_{1}}}
$$

for $|k| \leq 10$, and

$$
\begin{aligned}
& (1+|k|)^{s r} \int_{0}^{\infty}\left\|\pi_{k} e^{i t|\Delta|^{\alpha / 2}} f\right\|_{L^{p_{2}}}^{r} d t \\
& \leq(1+|k|)^{s r}\left\|\pi_{k} f\right\|_{L^{p_{1}}}^{r}|k|^{(\alpha-2) r n\left(1 / 2-1 / p_{2}\right)} \\
& \quad \times \int_{|k|^{2-\alpha}}^{\infty} t^{-r n\left(1 / 2-1 / p_{2}\right)} d t \\
& \quad+(1+|k|)^{s r}\left\|\pi_{k} f\right\|_{L^{p_{1}}}^{r} \int_{|k|^{2-\alpha}}^{\infty} t^{-r n\left(1 / 2-1 / p_{2}\right)} d t \\
& \leq(1+|k|)^{s r}\left\|\pi_{k} f\right\|_{L^{p_{1}}}^{r}\langle k\rangle^{(\alpha-2)} .
\end{aligned}
$$

This shows that

$$
\begin{aligned}
& \|\| e^{i t|\Delta|^{\alpha / 2}} f\left\|_{M_{p_{2}, q}^{s}\left(\mathbb{R}^{n}\right)}\right\|_{L^{r}(0, \infty)} \\
& \quad \leq\left\{\sum_{k}(1+|k|)^{s r}\left\|\pi_{k} f\right\|_{L^{p_{1}}}^{r}\langle k\rangle^{(\alpha-2)}\right\}^{1 / r} \\
& =\|f\|_{\mu_{p_{1}, r}^{s+(2-\alpha) / r}\left(\mathbb{R}^{n}\right)} .
\end{aligned}
$$

The theorem is proved.

5.3. Schrödinger Equation. Consider the Cauchy problem of the linear free Schrödinger equation

$$
\begin{gathered}
i \partial_{t} u=\Delta_{x} u, \quad(t, x) \in \mathbb{R}_{+} \times \mathbb{R}^{n} \\
u(0, x)=u_{0}(x) .
\end{gathered}
$$

The formal solution to this equation is given by

$$
u(t, x)=\frac{1}{(2 \pi)^{n}} \int_{\mathbb{R}^{n}} e^{i t|\xi|^{2}} \widehat{u_{0}}(\xi) e^{2 \pi i \xi \cdot x} d \xi=\left(e^{-i t \Delta} u_{0}\right)(x)
$$

By Theorem 1, we obtain the growth rate as $t \rightarrow \infty$ for the solution to the linear free Schrödinger equation.

Theorem 25. Let $u(t, x)$ be the solution of the above Cauchy problem of the Schrödinger equation. For $0<p \leq \infty$, one has

$$
\|u(t, \cdot)\|_{M_{p, q}^{s}\left(\mathbb{R}^{n}\right)} \preceq\left(1+t^{n(1 / p-1 / 2)}\right)\left\|u_{0}\right\|_{M_{p, q}^{s}\left(\mathbb{R}^{n}\right)},
$$

where the asymptotic factor $t^{n(1 / p-1 / 2)}$ is sharp as $t \rightarrow \infty$. 
5.4. Linear Cauchy Problem with Negative Power. We start with the following linear Cauchy problem with negative power:

$$
\begin{gathered}
i \partial_{t} u+\left|\Delta_{x}\right|^{-\alpha / 2} u=0, \quad(t, x) \in \mathbb{R}_{+} \times \mathbb{R}^{n}, \alpha>0, \\
u(0, x)=u_{0}(x) .
\end{gathered}
$$

The formal solution to this equation is given by

$$
u(t, x)=\frac{1}{(2 \pi)^{n}} \int_{\mathbb{R}^{n}} e^{i t|\xi|^{\alpha}} \widehat{u_{0}}(\xi) e^{2 \pi i \xi \cdot x} d \xi=\left(e^{-i t|\Delta|^{-\alpha / 2}} u_{0}\right)(x) .
$$

Proposition 26. Let $\alpha>0$ and $1 \leq p \leq 2$. One has

$$
\begin{aligned}
& \left\|\pi_{0} e^{-i t|\Delta|^{-\alpha / 2}} f\right\|_{L^{p}\left(\mathbb{R}^{n}\right)} \\
& \quad \preceq(1+t)^{([n / 2]+1)|2 / p-1|} \sum_{|j|_{\infty} \leq 1}\left\|\pi_{j} f\right\|_{H^{r}\left(\mathbb{R}^{n}\right)},
\end{aligned}
$$

where

$$
\frac{1}{r}>\frac{n+\alpha([n / 2]+1)(2-p)}{n p} .
$$

Proof. We only prove the case of odd $n$, since the proof for even $n$ is similar. For any fixed

$$
\beta>\alpha\left(\left[\frac{n}{2}\right]+1\right)
$$

we write

$$
\begin{aligned}
& \pi_{0} e^{-i t|\Delta|^{-\alpha / 2}} f(x) \\
& \quad=\frac{1}{(2 \pi)^{n}} \int_{\mathbb{R}^{n}} \sigma(\xi)|\xi|^{\beta} e^{i t|\xi|^{-\alpha}}|\xi|^{-\beta \widehat{f}}(\xi) e^{2 \pi i \xi \cdot x} d \xi \\
& \quad \simeq \pi_{0}|\Delta|^{\beta / 2} e^{-i t|\Delta|^{-\alpha / 2}} I_{\beta} f,
\end{aligned}
$$

where $I_{\beta}$ is the Riesz potential of order $\beta$. The kernel of $\pi_{0}|\Delta|^{\beta / 2} e^{-i t|\Delta|^{-\alpha / 2}}$ is

$$
\Omega_{\alpha, \beta}(x)=\int_{\mathbb{R}^{n}}|\xi|^{\beta} e^{i t|\xi|^{-\alpha}} \sigma(\xi) e^{2 \pi i \xi \cdot x} d \xi
$$

We first show

$$
\left\|\Omega_{\alpha, \beta} * f\right\|_{L^{1}} \preceq(1+t)^{\left[\frac{n}{2}\right]+1}\|f\|_{L^{1}} .
$$

To this end, by Young's inequality, we need to show

$$
\left\|\Omega_{\alpha, \beta}\right\|_{L^{1}\left(\mathbb{R}^{n}\right)} \preceq(1+t)^{[n / 2]+1} .
$$

It suffices to show the case $t \geq 1$. As the same argument in the proof of Theorem 1, with the Schwarz inequality we have

$$
\left\|\Omega_{\alpha, \beta}\right\|_{L^{1}\left(\mathbb{R}^{n}\right)} \preceq t^{n / 2}+\left.\int_{|x|>t}\left|\int_{\mathbb{R}^{n}}\right| \xi\right|^{\beta} e^{i t|\xi|^{-\alpha}} \sigma(\xi) e^{2 \pi i \xi \cdot x} d \xi \mid d x .
$$

Performing integration by parts $(n+1) / 2$ times on the second term, without loss of generality, we may write

$$
\begin{aligned}
& \left.\int_{|x|>t}\left|\int_{\mathbb{R}^{n}}\right| \xi\right|^{\beta} e^{i t|\xi|^{-\alpha}} \sigma(\xi) e^{2 \pi i \xi \cdot x} d \xi \mid d x \\
& \preceq t^{(n+1) / 2} \int_{|x|>t} \frac{1}{|x|^{(n+\varepsilon) / 2}} \\
& \quad \times\left|\frac{1}{|x|^{(1-\varepsilon) / 2}} \int_{\mathbb{R}^{n}} \psi(\xi) e^{i t|\xi|^{-\alpha}} \widetilde{\sigma}(\xi) e^{2 \pi i \xi \cdot x} d \xi\right| d x
\end{aligned}
$$

where $\widetilde{\sigma}(\xi)$ is a $C^{\infty}$ function supported in $[-1,1]^{n}$ and $\psi(\xi)$ is a function satisfying

$$
|\psi(\xi)| \preceq|\xi|^{\beta-((n+1) \alpha / 2)-((n+1) / 2)} .
$$

Choose a small $\varepsilon>0$ such

$$
2 \beta-\alpha(n+1)-\varepsilon>0 .
$$

Thus by Schwarz's inequality and the Pitt's theorem, we obtain

$$
\begin{aligned}
& \left.\int_{|x|>t}\left|\int_{\mathbb{R}^{n}}\right| \xi\right|^{\beta} e^{i t|\xi|^{-\alpha}} \sigma(\xi) e^{2 \pi i \xi \cdot x} d \xi \mid d x \\
& \preceq t^{(n+1) / 2}\left(\int_{\mathbb{R}^{n}} \mid \frac{1}{|x|^{(1-\varepsilon) / 2}} \int_{\mathbb{R}^{n}} \psi(\xi) e^{i t|\xi|^{-\alpha}} \widetilde{\sigma}(\xi)\right. \\
& \left.\times\left. e^{2 \pi i \xi \cdot x} d \xi\right|^{2} d x\right)^{1 / 2} \\
& t^{(n+1) / 2}\left(\int_{\mathbb{R}^{n}}|\widetilde{\sigma}(\xi)|^{2}|\xi|^{-n-\varepsilon}|\xi|^{2 \beta-\alpha(n+1)} d \xi\right)^{1 / 2} \preceq t^{(n+1) / 2} .
\end{aligned}
$$

Combining these estimates, we have

$$
\left\|\pi_{0} e^{-i t|\Delta|^{-\alpha / 2}} f\right\|_{L^{1}} \preceq t^{(n+1) / 2}\left\|I_{\beta} f\right\|_{L^{1}} \preceq t^{(n+1) / 2}\|f\|_{L_{-\beta}^{1}},
$$

where $L_{-\beta}^{1}$ denotes the $L^{1}$ Sobolev space of order $-\beta$. On the other hand, we have the easy energy estimate

$$
\left\|\pi_{0} e^{-i t|\Delta|^{-\alpha / 2}} f\right\|_{L^{2}} \preceq\|f\|_{L^{2}} \text {. }
$$

An interpolation yields that for all $1 \leq p \leq 2$,

$$
\left\|\pi_{0} e^{-i t|\Delta|^{-\alpha / 2}} f\right\|_{L^{p}} \preceq t^{(n+1)\left(1 / p^{-1 / 2)}\right.}\|f\|_{L_{-\gamma}^{p}},
$$

where

$$
\gamma=\beta\left(\frac{2}{p}-1\right)
$$

We now use the Sobolev imbedding theorem and the almost orthogonality of $\left\{\pi_{k}\right\}$ to obtain

$$
\left\|\pi_{0} e^{-i t|\Delta|^{-\alpha / 2}} f\right\|_{L^{p}\left(\mathbb{R}^{n}\right)} \preceq t^{(n+1)(1 / p-1 / 2)} \sum_{|j|_{\infty} \leq 1}\left\|\pi_{j} f\right\|_{H^{r}\left(\mathbb{R}^{n}\right)}
$$


with

$$
\frac{1}{r}=\frac{1}{p}+\frac{\beta(2 / p-1)}{n}=\frac{n+\beta(2-p)}{n p} .
$$

Since $\beta$ is an arbitrary number larger than $\alpha([n / 2]+1)$, the proposition now follows from Lemma 13.

Lemma 27. Let $\alpha>0$ and $2 \leq p \leq \infty$. One has

$$
\left\|\pi_{0} e^{-i t|\Delta|^{-\alpha / 2}} f\right\|_{L^{2}\left(\mathbb{R}^{n}\right)} \leq \sum_{|j|_{\infty} \leq 1}\left\|\pi_{j} f\right\|_{H^{r}\left(\mathbb{R}^{n}\right)},
$$

for any $r \leq 2$.

Proof. The almost orthogonality (Identity (46)) and the energy estimate give

$$
\left\|\pi_{0} e^{-i t|\Delta|^{-\alpha / 2}} f\right\|_{L^{2}\left(\mathbb{R}^{n}\right)} \leq \sum_{|j|_{\infty} \leq 1}\left\|\pi_{j} f\right\|_{L^{2}\left(\mathbb{R}^{n}\right)} .
$$

Thus the lemma follows from Lemma 13.

Now we are ready to give the proof of Theorem 5 .

Proof. The proof of (i) can be obtained from Propositions 18 and 26, and the definition and the modulation spaces. Similarly, the proof of (ii) follows by Proposition 18, Lemma 27 and the definition of the modulation spaces.

5.5. Nonlinear Cauchy Problem with Negative Power. Now, we study the following Cauchy problem of the nonlinear dispersive equations (NDE):

$$
\begin{gathered}
i \frac{\partial u}{\partial t}-|\Delta|^{-\alpha / 2} u+F(u)=0, \\
u(0, x)=u_{0}(x),
\end{gathered}
$$

where $F(u)=|u|^{2 k} u$ for some positive integer $k$. For $T>0$, the space $Y=C\left([0, T], M_{p, 1}^{s}\right)$ is defined by

$$
\begin{aligned}
C & \left([0, T], M_{p, 1}^{s}\right) \\
& =\left\{u(t, x):\|u\|_{C\left([0, T], M_{p, 1}^{s}\right)}=\sup _{0 \leq t \leq T}\|u(t, \cdot)\|_{M_{p, 1}^{s}}<\infty\right\} .
\end{aligned}
$$

Our proof will follow the same method used in [8], or, more precisely, the idea introduced in an earlier paper [13].

Now we give the proof of Theorem 6 .

Proof. In the proof, the letters $C_{j}, j=1,2,3$ denote some positive constants that are independent of all essential variables. We write the Cauchy problem in the equivalent form

$$
u(t, \cdot)=e^{\mathrm{i} t|\Delta|^{-\alpha / 2}} u_{0}-i \int_{0}^{t} e^{i(t-\tau)|\Delta|^{-\alpha / 2}} F(u(\tau, \cdot)) d \tau,
$$

and consider the mapping

$$
\mathfrak{\Im} u=e^{i t|\Delta|^{-\alpha / 2}} u_{0}-i \int_{0}^{t} e^{i(t-\tau)|\Delta|^{-\alpha / 2}} F(u(\tau, \cdot)) d \tau .
$$

We want to show that $\mathfrak{I}$ is a contraction.

By Theorem 5 and Lemma 12,

$$
\begin{aligned}
& \left\|e^{i t|\Delta|^{-\alpha / 2}} u_{0}\right\|_{Y} \\
& \quad \leq C_{1}(1+T)^{([n / 2]+1)|2 / p-1|}\left\|u_{0}\right\|_{M_{r, 1}^{s-\gamma}\left(\mathbb{R}^{n}\right)}+C_{1}\left\|u_{0}\right\|_{M_{p, 1}^{s}\left(\mathbb{R}^{n}\right)} .
\end{aligned}
$$

By Lemma 16, there is a constant $A_{2 k+1}>0$ for which we have

$$
\left\||u(t, \cdot)|^{2 k} u(t, \cdot)\right\|_{M_{r, 1}^{s}} \leq A_{2 k+1}\|u(t, \cdot)\|_{M_{(2 k+1) r, 1}^{2 k+1}}^{2 k} .
$$

Thus, by Theorem 5 and Lemma 12,

$$
\begin{gathered}
\left\|\int_{0}^{t} e^{i(t-\tau)|\Delta|^{-\alpha / 2}} F(u(\tau, \cdot)) d \tau\right\|_{C\left([0, T], M_{p, 1}^{s}\right)} \\
\leq \sup _{0<t \leq T} \int_{0}^{t}\left\|e^{i(t-\tau)|\Delta|^{-\alpha / 2}} F(u(\tau, \cdot))\right\|_{M_{p, 1}^{s}} d \tau \\
\leq C_{1} \sup _{0<t \leq T} \int_{0}^{t}(1+(t-\tau))^{([n / 2]+1)|2 / p-1|} \\
\quad \times\left\||u(\tau, \cdot)|^{2 k} u(\tau, \cdot)\right\|_{M_{r, 1}^{s}} d \tau \\
\leq C_{2} M_{k} T(1+T)^{([n / 2]+1)|2 / p-1|} \sup _{0 \leq t \leq T}\|u(t, \cdot)\|_{M_{r(2 k+1), 1}^{s+1}}^{2 k+1} \\
\leq C_{2} M_{k} T(1+T)^{([n / 2]+1)|2 / p-1|} \sup _{0 \leq t \leq T}\|u(t, \cdot)\|_{M_{p, 1}^{s}}^{2 k+1} .
\end{gathered}
$$

The last inequality is because

$$
r(2 k+1) \geq p
$$

and the imbedding relation $M_{p, 1}^{s} \subset M_{r(2 k+1), 1}^{s}$.

As a consequence,

$$
\begin{aligned}
& \|\Im \mathfrak{J}\|_{C\left([0, T], M_{p, 1}^{s}\right)} \\
& =\sup _{0 \leq t \leq T}\|\Im u(t, \cdot)\|_{M_{p, 1}^{s}} \\
& \leq C_{3}(1+T)^{([n / 2]+1)|2 / p-1|} \\
& \quad \times\left(\left\|u_{0}\right\|_{M_{r, 1}^{s-\gamma}\left(\mathbb{R}^{n}\right)}+T \sup _{0 \leq t \leq T}\|u(t, \cdot)\|_{M_{p, 1}^{s}}^{2 k+1}\right) \\
& \quad+C_{1}\left\|u_{0}\right\|_{M_{p, 1}^{s}\left(\mathbb{R}^{n}\right)} .
\end{aligned}
$$

Fix an $L>0$ such that

$$
C_{3}\left\|u_{0}\right\|_{M_{r, 1}^{s-\gamma}\left(\mathbb{R}^{n}\right)}+C_{1}\left\|u_{0}\right\|_{M_{p, 1}^{s}\left(\mathbb{R}^{n}\right)}<\frac{L}{4} .
$$


Let $B_{L}$ be the closed ball of radial $L$ centered at the origin in the space $C\left([0, T], M_{p, 1}^{s}\right)$. Now we choose $T_{0}$ such that for any $u \in B_{L}$ and $T \in\left(0, T_{0}\right]$,

$$
\begin{aligned}
\| \Im & u \|_{C\left([0, T], M_{p, 1}^{s}\right)} \\
\leq & C_{3}(1+T)^{([n / 2]+1)|2 / p-1|}\left(\left\|u_{0}\right\|_{M_{r, 1}^{s-\gamma}\left(\mathbb{R}^{n}\right)}+T L^{2 k+1}\right) \\
& +C_{1}\left\|u_{0}\right\|_{M_{p, 1}^{s}\left(\mathbb{R}^{n}\right)} \leq L .
\end{aligned}
$$

By this choice, $\mathfrak{\Im}$ is a mapping form $B_{L}$ into $B_{L}$.

Furthermore, an easy computation gives that

$$
\begin{aligned}
\| \Im u & -\Im v \|_{C\left([0, T], M_{p, 1}^{s}\right)} \\
\leq & C_{3}(1+T)^{([n / 2]+1)|2 / p-1|} \\
& \times T \sup _{0 \leq t \leq T}\|u(t, \cdot)-v(t, \cdot)\|_{M_{p, 1}^{s}}(2 k+1) L^{2 k} \\
\leq & \frac{1}{2}\|u-v\|_{C\left([0, T], M_{p, 1}^{s}\right)},
\end{aligned}
$$

for all $T \in\left(0, T_{0}\right]$ if $T_{0}$ is suitably chosen. Finally, using the Banach contraction mapping theorem, we obtain a fixed point in $B_{L}$. This is the solution of the Cauchy problem. We prove the theorem.

Using a similar argument with the help of (ii) in Theorem 5, we can prove Theorem 7 .

\section{Acknowledgment}

This work is partially supported by the NSF of China (Grants nos. 10931001, 10871173, 11201103).

\section{References}

[1] A. Miyachi, F. Nicola, S. Rivetti, A. Tabacco, and N. Tomita, "Estimates for unimodular Fourier multipliers on modulation spaces," Proceedings of the American Mathematical Society, vol. 137, no. 11, pp. 3869-3883, 2009.

[2] H. G. Feichtinger, "Modulation spaces on locally compact abeliangroups," Tech. Rep., University of Vienna, 1983.

[3] M. Krishna, R. Radha, and S. Thangavelu, Eds., Wavelets and Their Applications, Allied Publishers, New Delhi, India, 2003.

[4] H. G. Feichtinger, "Modulation spaces: looking back and ahead," Sampling Theory in Signal and Image Processing, vol. 5, no. 2, pp. 109-140, 2006.

[5] K. Gröchenig, Foundations of Time-Frequency Analysis, Applied and Numerical Harmonic Analysis, Birkhäuser, Boston, Mass, USA, 2001.

[6] J. Sjöstrand, "An algebra of pseudodifferential operators," Mathematical Research Letters, vol. 1, no. 2, pp. 185-192, 1994.

[7] J. Toft, "Continuity properties for modulation spaces, with applications to pseudo-differential calculus. II," Annals of Global Analysis and Geometry, vol. 26, no. 1, pp. 73-106, 2004.

[8] A. Bényi and K. A. Okoudjou, "Local well-posedness of nonlinear dispersive equations on modulation spaces," Bulletin of the London Mathematical Society, vol. 41, no. 3, pp. 549-558, 2009.
[9] A. Bényi, K. Gröchenig, K. A. Okoudjou, and L. G. Rogers, "Unimodular Fourier multipliers for modulation spaces," Journal of Functional Analysis, vol. 246, no. 2, pp. 366-384, 2007.

[10] E. Cordero and F. Nicola, "Remarks on Fourier multipliers and applications to the wave equation," Journal of Mathematical Analysis and Applications, vol. 353, no. 2, pp. 583-591, 2009.

[11] J. Chen, D. Fan, and L. Sun, "Asymptotic estimates for unimodular Fourier multipliers on modulation spaces," Discrete and Continuous Dynamical Systems A, vol. 32, no. 2, pp. 467-485, 2012.

[12] L. Sun, "Mixed norm estimates on modulation spaces for certain unimodular multipliers," Panamerican Mathematical Journal, vol. 21, no. 2, pp. 75-90, 2011.

[13] B. Wang, L. Zhao, and B. Guo, "Isometric decomposition operators, function spaces $E_{p, q}^{\lambda}$ and applications to nonlinear evolution equations," Journal of Functional Analysis, vol. 233, no. 1, pp. 1-39, 2006.

[14] B. Wang and H. Hudzik, "The global Cauchy problem for the NLS and NLKG with small rough data," Journal of Differential Equations, vol. 232, no. 1, pp. 36-73, 2007.

[15] N. Tomita, "Unimodular Fourier multipliers on modulation spaces $M^{p, q}$ for $0<p<1$," in Harmonic Analysis and Nonlinear Partial Differential Equations, RIMS Kkyroku Bessatsu, B18, pp. 125-131, Research Institute for Mathematical Sciences (RIMS), Kyoto, Japan, 2010.

[16] J. Chen and D. Fan, "Estimates for wave and Klein-Gordon equations on modulation spaces," Science China, vol. 55, no. 10, pp. 2109-2123, 2012.

[17] B. Wang and C. Huang, "Frequency-uniform decomposition method for the generalized BO, KdV and NLS equations," Journal of Differential Equations, vol. 239, no. 1, pp. 213-250, 2007.

[18] B. Wang, C. Hao, and C. Huo, Harmonic Analysis Methods for Nonlinear Evolution Equations, World Scientific, 2011.

[19] E. M. Stein, Beijing Lectures in Harmonic Analysis, Annals of Mathematics Studies, vol. 112, Princeton University Press, Princeton, NJ, USA, 1982.

[20] H. Triebel, Theory of Function Spaces, vol. 78 of Monographs in Mathematics, Birkhäuser, Basel, Switzerland, 1983.

[21] A.-P. Calderón and A. Torchinsky, "Parabolic maximal functions associated with a distribution. II," Advances in Mathematics, vol. 24, no. 2, pp. 101-171, 1977.

[22] M. Kobayashi, "Modulation spaces $M^{p, q}$ for $0<p, q \leq \infty$," Journal of Function Spaces and Applications, vol. 4, no. 3, pp. 329-341, 2006.

[23] S. Wainger, "Special trigonometric series in $k$-dimensions," Memoirs of the American Mathematical Society, vol. 59, article 102, 1965.

[24] A. Miyachi, “On some singular Fourier multipliers," Journal of the Faculty of Science, vol. 28, no. 2, pp. 267-315, 1981. 


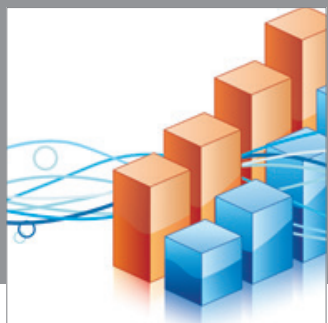

Advances in

Operations Research

mansans

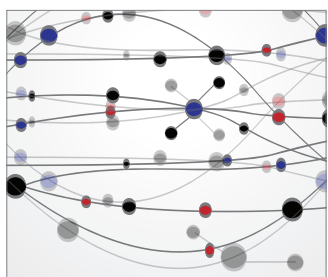

The Scientific World Journal
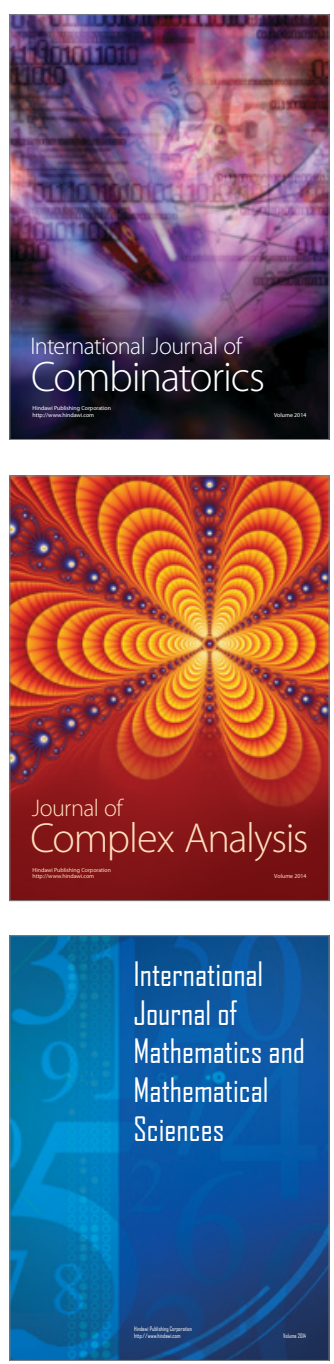
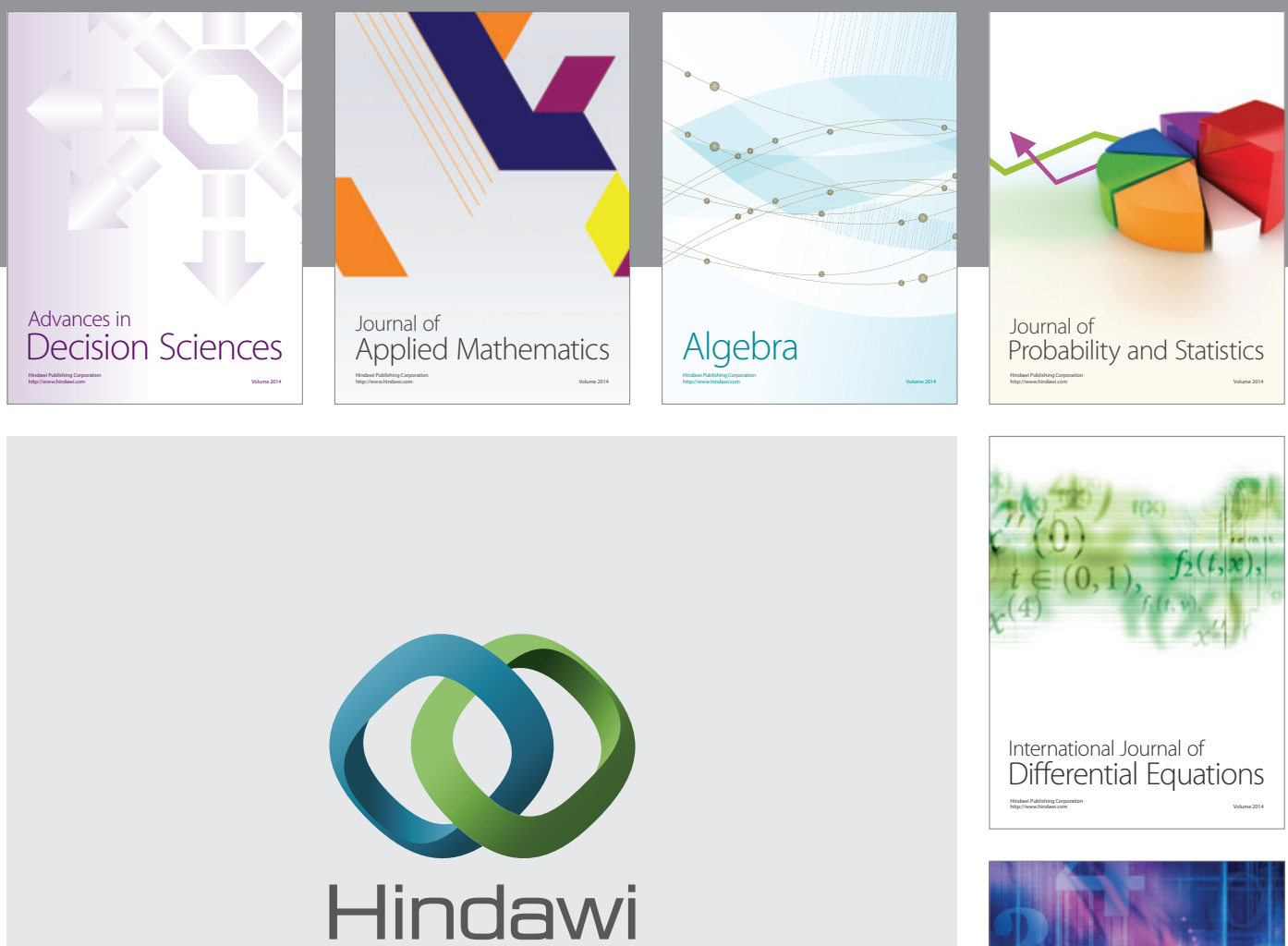

Submit your manuscripts at http://www.hindawi.com
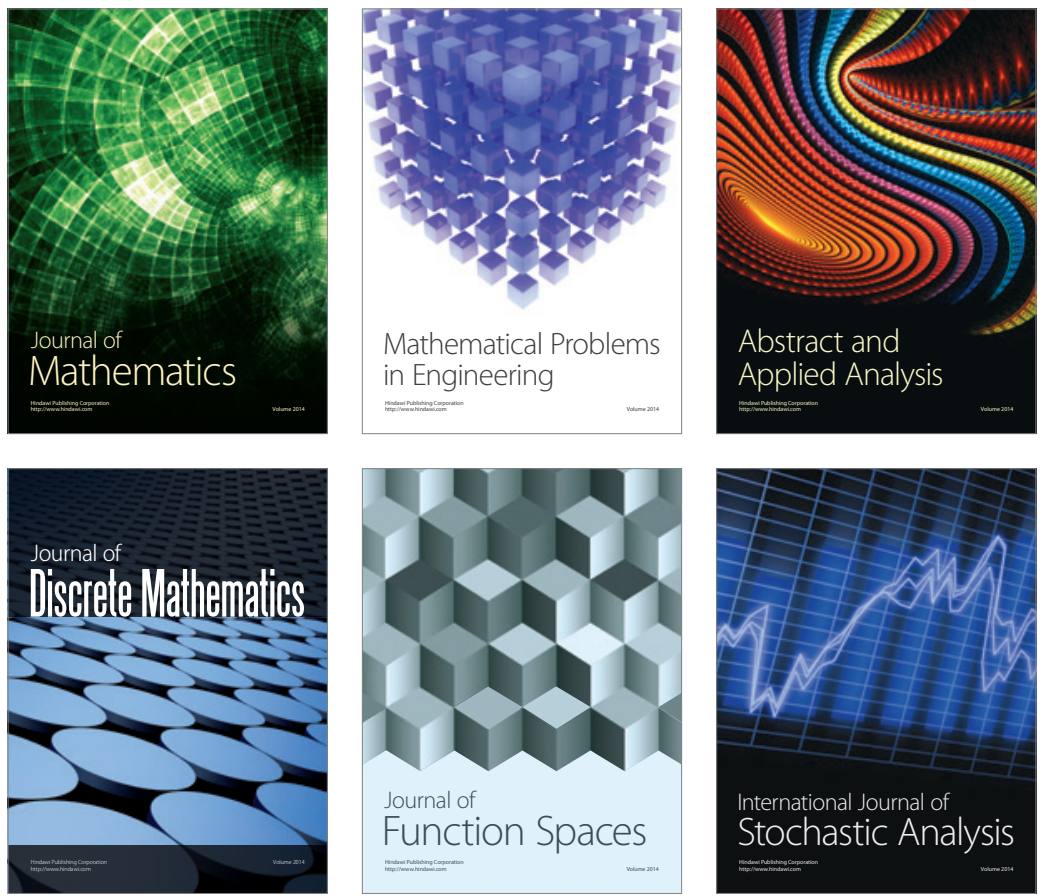

Journal of

Function Spaces

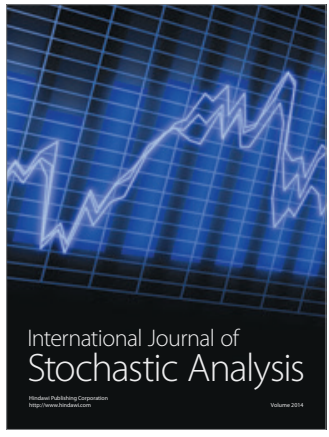

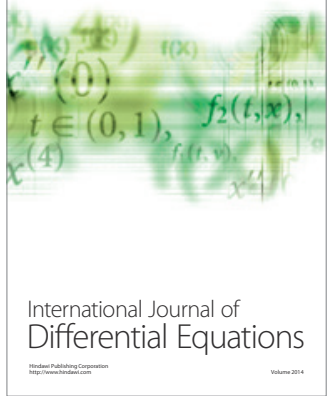
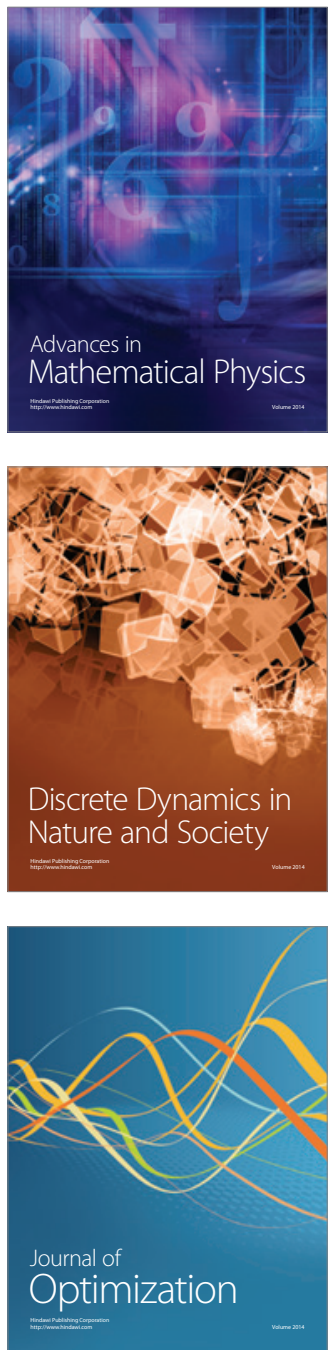